\title{
Convergent Synthesis of the Tricyclic Core of the Dictyosphaeric
}

\section{Acids}

Christopher W. Barfoot, ${ }^{\mathrm{a}}$ Alan R. Burns, ${ }^{\mathrm{a}}$ Michael G. Edwards, ${ }^{\mathrm{a}}$ Martin Kenworthy,

Mahmood Ahmed, ${ }^{\mathrm{b}}$ Stephen S. Shanahan, ${ }^{\mathrm{c}}$ and Richard J. K. Taylor ${ }^{\mathrm{a}^{*}}$

${ }^{a}$ Department of Chemistry, University of York, Heslington, York YO10 5DD

${ }^{\mathrm{b}}$ GlaxoSmithKline, New Frontiers Science Park (North), Third Avenue, Harlow, Essex CM19 5AW, UK

${ }^{\mathrm{c}}$ GlaxoSmithKline, Chemical Development, Old Powder Mills, Tonbridge, Kent, TN11 $9 A N, U K$

\section{Supporting Information}

\section{General Methods}

All reagents were purchased from commercial sources and used without further purification, unless otherwise stated. All reactions were carried out in oven-dried or flame-dried glassware under a nitrogen or argon atmosphere using standard syringe and septum techniques unless otherwise stated. DME and THF were freshly distilled from sodium/benzophenone and dichloromethane from calcium hydride, immediately prior to use. Toluene was dried using a MBraun Solvent Purification System. Thin layer chromatography was performed on precoated $0.2 \mathrm{~mm}$ Merck Kieselgel $60 \mathrm{~F}_{254}$ silica plates and compounds were visualized under $245 \mathrm{~nm}$ ultraviolet irradiation followed by staining in either alkaline potassium permanganate or ethanolic vanillin solution. Flash column chromatography was performed using Fluka Kieselgel $60 \mathrm{~F}$ (220-440 mesh) with the indicated solvents. Petroleum ether refers to the fraction with boiling range $40-60{ }^{\circ} \mathrm{C}$.

${ }^{1} \mathrm{H}$ and ${ }^{13} \mathrm{C}$ NMR spectra were obtained using either a JEOL $400 \mathrm{MHz}$ spectrophotometer operating at either $400 \mathrm{MHz}$ or $100 \mathrm{MHz}$ or a Bruker AMX 500 spectrometer operating at $500 \mathrm{MHz}$ or $125 \mathrm{MHz}$, respectively. Data are expressed in parts per million relative to residual solvent. All $J$ values are given in $\mathrm{Hz}$. 
Assignments are made with the aid of DEPT 135, COSY, HSQC and nOe experiments. Optical rotations were measured using a Jasco DIP 370 Digital Polarimeter at $\lambda=598 \mathrm{~nm}$ and are given in units of $10^{-1} \mathrm{deg} \mathrm{cm}^{2} \mathrm{~g}^{-1}$. Infrared spectra were recorded with a ThermoNicolet IR100 spectrophotometer as thin films between sodium chloride plates. Absorption maxima are expressed in wavenumbers $\left(\mathrm{cm}^{-1}\right)$.

Low resolution electrospray ionisation (ESI) mass spectra were recorded on a Kratos MS 25 spectrometer. Chemical ionisation (CI) and high resolution mass spectra were recorded on a Micromass Autospec spectrometer. 


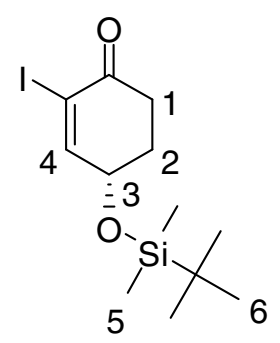

To a stirred solution of enone 17 (1.214 g, $5.36 \mathrm{mmol}, 1.00$ eq.) in THF/ $\mathrm{H}_{2} \mathrm{O}$ (1:1, 16 $\mathrm{mL}$ ) was added DMAP (0.131 g, $1.07 \mathrm{mmol}, 0.2$ eq.) and $\mathrm{K}_{2} \mathrm{CO}_{3}(1.111 \mathrm{~g}, 8.04$ mmol, 1.5 eq.). The resulting solution was stirred for 5 min before iodine crystals $(2.042 \mathrm{~g}, 8.04 \mathrm{mmol}, 1.50 \mathrm{eq}$.) were added and the reaction stirred in the dark for $6 \mathrm{~h}$, when the reaction was judged to be complete by TLC analysis. The reaction was diluted with EtOAc $(50 \mathrm{~mL})$ and the organic phase washed with sat. $\mathrm{Na}_{2} \mathrm{~S}_{2} \mathrm{O}_{3}$ (aq.) solution $(80 \mathrm{~mL})$ and $10 \% \mathrm{HCl}$ (aq.) solution $(80 \mathrm{~mL})$. The $\mathrm{HCl}$ (aq.) phase was extracted with EtOAc $(3 \times 50 \mathrm{~mL})$ and the combined organic phases dried $\left(\mathrm{Na}_{2} \mathrm{SO}_{4}\right)$ and concentrated in vacuo. The resulting crude product was purified by flash column chromatography on silica gel (eluent: 9:1 Pet. ether/EtOAc) to give the title compound $18(1.527 \mathrm{~g}, 81 \%)$ as an orange oil, $R_{f} 0.34(9: 1$ Pet. ether/EtOAc $) ;[\alpha]^{22}{ }_{\mathrm{D}}=-60.5(c=$ 1.52, $\left.\mathrm{CHCl}_{3}\right)$, lit. ${ }^{1}[\alpha]^{22}{ }_{\mathrm{D}}=-44.4\left(c=1.68, \mathrm{CH}_{2} \mathrm{Cl}_{2}\right) ;{ }^{1} \mathrm{H} \mathrm{NMR}\left(400 \mathrm{MHz}, \mathrm{CDCl}_{3}\right): \delta$ 7.61 (1H, dd, $J=3.0,1.5, \mathrm{H}-4), 4.51$ (1H, ddd, $J=9.0,5.0,3.0, \mathrm{H}-3), 2.84$ (1H, ddd, $J=17.0,4.5,4.5, \mathrm{H}-1 \mathrm{a}), 2.50$ (1H, ddd, $J=17.0,12.5,4.5, \mathrm{H}-1 \mathrm{~b}), 2.25$ (1H, dddd, $J=$ 14.0, 9.0, 4.5, 1.5, H-2a), 2.03 (1H, m, H-2b), 0.91 (9H, s, H-6), 0.12 (6H, 2 x s, H-5). ${ }^{1} \mathrm{H}$ NMR data consistent with those reported in the literature. ${ }^{1}$ 


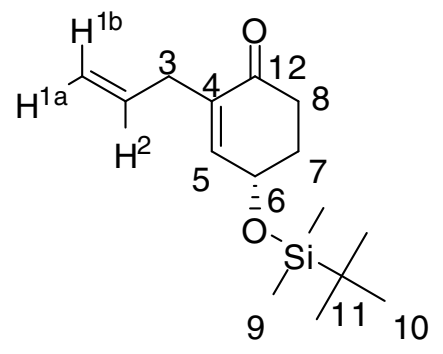

To allyltributylstannane $(2.851 \mathrm{~g}, 8.61 \mathrm{mmol}, 2.0$ eq. $)$ and $\mathrm{Pd}\left(\mathrm{PPh}_{3}\right)_{4}(0.373 \mathrm{~g}, 0.32$ mmol, 0.075 eq.) was added iodoenone 18 (1.517 g, 4.31 mmol, 1.0 eq.) in dry, argon sparged THF $(30 \mathrm{~mL})$. The system was purged via $10 \mathrm{x}$ vacuum/argon cycles and then maintained under an argon atmosphere. The reaction mixture was heated to reflux and stirred for $17 \mathrm{~h}$, when judged to be complete by TLC analysis. After cooling to rt, the reaction mixture was concentrated in vacuo and the resulting crude product was purified by flash column chromatography on 10\% KF / silica gel (eluent: 19:1 Pet. ether/EtOAc) to give the title compound $19(0.948 \mathrm{~g}, 83 \%)$ as a pale-yellow oil; $R_{f}$ 0.37 (9:1 Pet. ether/EtOAc); $[\alpha]_{\mathrm{D}}^{22}=-69.3\left(c=1.29, \mathrm{CHCl}_{3}\right) ; v_{\max }(\mathrm{film}) / \mathrm{cm}^{-1} 2954$, $2930(\mathrm{CH}), 1681(\mathrm{C}=\mathrm{O}) ;{ }^{1} \mathrm{H}$ NMR $\left(400 \mathrm{MHz}, \mathrm{CDCl}_{3}\right): \delta 6.56(1 \mathrm{H}, \mathrm{dt}, J=3.0,1.5, \mathrm{H}-$ 5), 5.85-5.74 (1H, m, H-2), 5.09-5.07 (1H, m, H-1a or H-1b), 5.06-5.03 (1H, m, H-1a or H-1b), 4.52 (1H, ddd, $J=11.5,4.5,1.5, \mathrm{H}-6), 2.93$ (2H, m, H-3), 2.59 (1H, ddd, $J$ $=17.0,4.5,4.5, \mathrm{H}-8 \mathrm{a}), 2.33$ (1H, ddd, $J=17.0,13.0,4.5, \mathrm{H}-8 \mathrm{~b}), 2.19$ (1H, dddd. $J=$ 13.0, 9.5, 4.5, 1.5, H-7a), 2.01-1.90 (1H, m, H-7b), 0.91 (9H, s, H-10), 0.11 (6H, 2 x s, H-9); ${ }^{13} \mathrm{C}$ NMR (100 MHz, $\mathrm{CDCl}_{3}$ ): $\delta 198.4$ (C-12), 149.3 (C-5), 137.3 (C-4), 135.3 (C-2), 117.0 (C-1), 67.6 (C-6), 35.9 (C-7), 33.4 (C-8), 25.9 (C-10), 18.3 (C-11), -4.41 (C-9a), -4.54 (C-9b); m/z (ESI) $267[\mathrm{MH}]^{+}$; HRMS (ESI): calcd. for $\mathrm{C}_{15} \mathrm{H}_{27} \mathrm{O}_{2} \mathrm{Si}$, 267.1775. Found: $[\mathrm{MH}]^{+}, 267.1778$ (1.2 ppm error). 


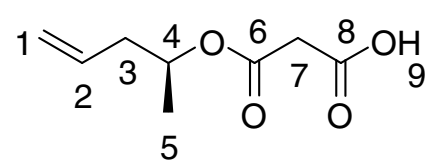

To a stirred solution of malonic acid (0.483 g, $4.64 \mathrm{mmol}, 2.0$ eq.) in $\mathrm{MeCN}$ (20 mL) was added DMAP $(0.057 \mathrm{~g}, 0.46 \mathrm{mmol}, 0.2$ eq. $)$. After cooling to $0^{\circ} \mathrm{C}$, $(S)-(+)-4-$ penten-2-ol (0.24 mL, $2.32 \mathrm{mmol}, 1.0$ eq.) was added, followed by DCC (1.005 g, $4.87 \mathrm{mmol}, 2.1$ eq.) in $\mathrm{MeCN}(10 \mathrm{~mL})$, over a period of $10 \mathrm{~min}$. The resulting suspension was stirred at $0^{\circ} \mathrm{C}$ for $10 \mathrm{~min}$, warmed to $\mathrm{rt}$ and stirred for $4 \mathrm{~h}$, when the reaction was judged to be complete by TLC analysis. The precipitate was filtered off and the filter cake washed with $\mathrm{MeCN}(100 \mathrm{~mL})$. The filtrate was concentrated in vacuo and the resulting crude product dissolved in EtOAc $(25 \mathrm{~mL})$ and washed with $\mathrm{H}_{2} \mathrm{O}(3 \times 25 \mathrm{~mL})$ and sat. $\mathrm{NaHCO}_{3}$ (aq.) solution (4 x $\left.25 \mathrm{~mL}\right)$. The combined aqueous phases were acidified carefully with sat. $\mathrm{NaH}_{2} \mathrm{PO}_{4}$ (aq.) solution $(60 \mathrm{~mL})$ and taken to pH 3 with $10 \% \mathrm{HCl}$ (aq.) solution. This acidified aqueous phase was extracted with EtOAc $(4 \times 25 \mathrm{~mL})$ and the combined organic phases dried $\left(\mathrm{Na}_{2} \mathrm{SO}_{4}\right)$ and concentrated in vacuo to give the title compound $20(0.398 \mathrm{~g}, 99 \%)$ as a red oil, $R_{f}$ $0.11(3: 1$ Pet. ether/EtOAc +2 drops AcOH $) ;[\alpha]_{\mathrm{D}}^{22}=-13.1\left(c=0.97, \mathrm{CHCl}_{3}\right) ; v_{\max }$ (film)/cm $3504(\mathrm{OH}), 3080\left(\mathrm{CH}_{\text {alkene }}\right), 2982,2938(\mathrm{CH}), 1736(\mathrm{C}=\mathrm{O}), 1643(\mathrm{C}=\mathrm{C})$; ${ }^{1} \mathrm{H} \mathrm{NMR}\left(400 \mathrm{MHz}, \mathrm{CDCl}_{3}\right): \delta$ 5.79-5.69 (1H, m, H-2), 5.12-5.04 (3H, m, H-1 and H4), 3.41 (2H, s, H-7), 2.41-2.28 (2H, m, H-3), 1.27 (3H, d, $J=6.5, \mathrm{H}-5) ;{ }^{13} \mathrm{C} \mathrm{NMR}$ (100 MHz, $\mathrm{CDCl}_{3}$ ): $\delta 170.5$ (C-6), 167.9 (C-8), 133.6 (C-2), 118.9 (C-1), 73.0 (C-4), 40.9 (C-3), 40.6 (C-7), $19.9(\mathrm{C}-5) ; m / z(\mathrm{CI}): 190\left(100 \%,\left[\mathrm{MNH}_{4}\right]^{+}\right)$.

HRMS: calcd. for $\mathrm{C}_{8} \mathrm{H}_{16} \mathrm{NO}_{4}, 190.1079$. Found: [MNH$]^{+}, 190.1077$ (1.2 ppm error). 


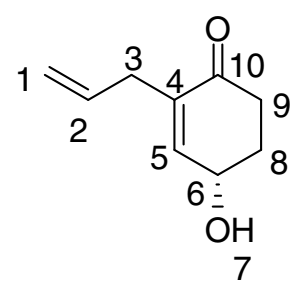

To a stirred solution of TBS-ether $19(0.448 \mathrm{~g}, 1.68 \mathrm{mmol}, 1.0$ eq. $)$ at $0^{\circ} \mathrm{C}$ in $\mathrm{THF}(11$ $\mathrm{mL}$ ), was added TBAF solution (2.02 $\mathrm{mL}$ of a 1.0 M solution in THF, $2.02 \mathrm{mmol}, 1.2$ eq.). The cooling bath was removed and the reaction stirred at $\mathrm{rt}$ for $5 \mathrm{~h}$, when judged to be complete by TLC analysis. The crude reaction mixture was adsorbed onto $\mathrm{SiO}_{2}$, filtered through a $5 \mathrm{~cm}$ pad of $\mathrm{SiO}_{2}$ (eluent 1:1 - 2:1 EtOAc/Pet. ether - 100\% EtOAc) and the filtrate concentrated in vacuo to give the title compound $19 b(0.265 \mathrm{~g}$, $104 \%)$ as a brown oil, which was taken onto the next step without further purification. A small sample was purified further by flash column chromatography on silica gel (eluent: 1:1 Pet. ether/EtOAc) to allow for full characterisation; $R_{f}=0.26$ $(1: 1 \mathrm{EtOAc} /$ Pet. ether $) ;[\alpha]_{\mathrm{D}}^{22}=-39.3\left(c=1.03, \mathrm{CHCl}_{3}\right) ; v_{\max }(\mathrm{film}) / \mathrm{cm}^{-1} 3400(\mathrm{OH})$, $2953(\mathrm{CH}), 1674(\mathrm{C}=\mathrm{O}) ;{ }^{1} \mathrm{H}$ NMR $\left(400 \mathrm{MHz}, \mathrm{CDCl}_{3}\right): \delta 6.66(1 \mathrm{H}, \mathrm{dt}, J=3.0,1.5, \mathrm{H}-$ 5), 5.84-5.74 (1H, m, H-2), 5.09-5.08 (1H, m, H-1a or H-1b), 5.06-5.04 (1H, m, H-1a or H-1b), 4.57 (1H, ddd, $J=11.5,4.5,1.5, \mathrm{H}-6), 2.94$ (2H, m, H-3), 2.61 (1H, ddd, $J$ $=17.0,4.5,4.5, \mathrm{H}-9 \mathrm{a}), 2.40-2.29(2 \mathrm{H}, \mathrm{m}, \mathrm{H}-9 \mathrm{~b}$ and $\mathrm{H}-8 \mathrm{a}), 1.99-1.89(1 \mathrm{H}, \mathrm{m}, \mathrm{H}-8 \mathrm{~b})$, 1.78 (1H, br s, H-7); ${ }^{13} \mathrm{C}$ NMR (100 MHz, $\left.\mathrm{CDCl}_{3}\right): \delta 198.6$ (C-10), 147.9 (C-5), 138.4 (C-4), 135.2 (C-4), 117.3 (C-1), 66.7 (C-6), 35.3 (C-8), 32.8 (C-3), 32.5 (C-9); m/z (ESI) $153[\mathrm{MH}]^{+}, 186[\mathrm{MNa}]^{+}$; HRMS (ESI): calcd. for $\mathrm{C}_{9} \mathrm{H}_{13} \mathrm{O}_{2}, 153.0910$. Found: $[\mathrm{MH}]^{+}, 153.0912$ (1.3 ppm error). 


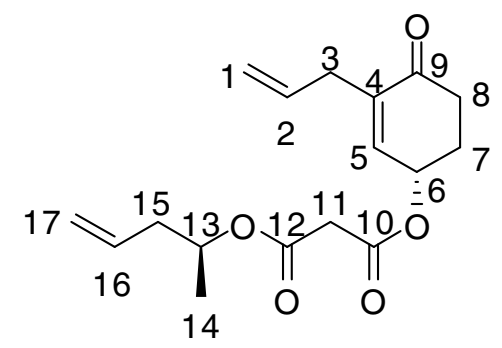

To a stirred solution of alcohol 19b and acid 20 (0.290 g, $1.68 \mathrm{mmol}, 1.0$ eq.) in dry toluene $(42 \mathrm{~mL})$, was added ${ }^{\mathrm{i}} \mathrm{Pr}_{2} \mathrm{NEt}(0.57 \mathrm{~mL}, 3.28 \mathrm{mmol}, 1.95$ eq.). After cooling to $0^{\circ} \mathrm{C}, \mathrm{T}^{\circledR} \mathrm{P}^{\circledR}[50 \% \mathrm{w} / \mathrm{w}$ in toluene $(1.392 \mathrm{~g}, 2.19 \mathrm{mmol}, 1.3 \mathrm{eq})]$ was added and the resulting solution stirred at $0^{\circ} \mathrm{C}$ for $10 \mathrm{~min}$, warmed to $\mathrm{rt}$ and stirred for $18 \mathrm{~h}$, when the reaction was judged to be complete by TLC analysis. The reaction mixture was diluted with EtOAc $(75 \mathrm{~mL})$ and the organic phase washed with $\mathrm{H}_{2} \mathrm{O}(2 \times 100 \mathrm{~mL})$, sat. $\mathrm{NaHCO}_{3}$ (aq.) $(100 \mathrm{~mL})$ and sat. brine (aq.) $(100 \mathrm{~mL})$. The combined organic phases were dried $\left(\mathrm{Na}_{2} \mathrm{SO}_{4}\right)$, concentrated in vacuo and the resulting crude product was purified by flash column chromatography on silica gel (eluent: 3:2 Pet. ether/ $\left.\mathrm{Et}_{2} \mathrm{O}\right)$ to give the title compound $21(0.438 \mathrm{~g}, 85 \%)$ as a colourless oil, $R_{f} 0.23$ (3:2 Pet. ether/ $\left.\mathrm{Et}_{2} \mathrm{O}\right) ;[\alpha]_{\mathrm{D}}^{22}=-86.4\left(c=1.03, \mathrm{CHCl}_{3}\right) ; v_{\max }(\mathrm{film}) / \mathrm{cm}^{-1} 2979,2937$ $(\mathrm{CH}), 1747,1730\left(\mathrm{C}=\mathrm{O}_{\text {ester }}\right), 1682\left(\mathrm{C}=\mathrm{O}_{\text {ketone }}\right) ;{ }^{1} \mathrm{H}$ NMR $\left(400 \mathrm{MHz}, \mathrm{CDCl}_{3}\right): \delta 6.57$ $(1 \mathrm{H}, \mathrm{dt}, J=3.0,1.5, \mathrm{H}-5), 5.81-5.68$ (2H, m, H-2 and H-16), $5.62(1 \mathrm{H}, \mathrm{m}, \mathrm{H}-6), 5.11-$ 4.98 (5H, m, H-1, H-17 and H-13), 3.38 (2H, s, H-11), 2.97-2.94 (2H, m, H-3), 2.64 (1H, ddd, $J=16.5,5.0,5.0, \mathrm{H}-8 \mathrm{a}), 2.47-2.24$ (4H, m, H-8b, H-15 and H-7a), 2.08 (1H, dddd, $J=13.0,11.5,8.5 .5 .0, \mathrm{H}-7 \mathrm{~b}), 1.23$ (3H, d, $J=6.5, \mathrm{H}-14) ;{ }^{13} \mathrm{C}$ NMR $(100$ $\mathrm{MHz}, \mathrm{CDCl}_{3}$ ): $\delta 197.7$ (C-9), 166.5 (C-12), 166.2 (C-10), 142.1 (C-5), 140.5 (C-4), 134.7 (C-2), 133.4 (C-16), 118.3 (C-1), 117.6 (C-17), 71.7 (C-6), 69.3 (C-13), 41.6 (C-11), 39.8 (C-15), 34.8 (C-3), 32.8 (C-8), 28.4 (C-7), 19.0 (C-14); m/z (ESI) 329 $[\mathrm{MNa}]^{+}, 307[\mathrm{MH}]^{+}$; HRMS (ESI): calcd. for $\mathrm{C}_{17} \mathrm{H}_{22} \mathrm{NaO}_{5}, 329.1359$. Found: [MNa ${ }^{+}$, 329.1355 (1.3 ppm error). 


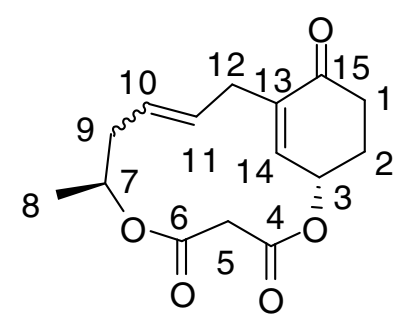

A stirred solution of diene 21 ( $0.100 \mathrm{~g}, 0.33 \mathrm{mmol}, 1.00$ eq.) in dry, argon sparged $\mathrm{CH}_{2} \mathrm{Cl}_{2}(145 \mathrm{~mL})$ was purged via $10 \times$ vacuum/argon cycles, maintained under an argon atmosphere and heated to reflux. Hoveyda-Grubbs catalyst, $2^{\text {nd }}$ generation (0.021 g, $0.033 \mathrm{mmol}, 0.10$ eq.) in $\mathrm{CH}_{2} \mathrm{Cl}_{2}(20 \mathrm{~mL})$ was added over $45 \mathrm{~min}$ via syringe pump and the resulting green/orange solution was stirred at reflux for $18 \mathrm{~h}$, when the reaction was judged to be complete by TLC analysis. After cooling to rt, the reaction mixture was filtered through a pad of silica, adsorbed onto $\mathrm{SiO}_{2}$ and purified by flash column chromatography on silica gel (eluent: 2:3 Pet. ether/EtOAc) to give the title compound $22(0.063 \mathrm{~g}, 69 \%)$ as a colourless oil as in a $3: 2$ mixture of $E / Z$ isomers, $R_{f}=0.36$ (2:3 Pet. ether/ $\left.\mathrm{Et}_{2} \mathrm{O}\right)$. A small sample was further purified by flash column chromatography on $\mathrm{AgNO}_{3} / \mathrm{SiO}_{2}{ }^{2}$ to separate the $E / Z$ isomers and aid spectral interpretation.

Major isomer (E), $R_{f} 0.24\left(\mathrm{AgNO}_{3}\right.$ doped $\mathrm{SiO}_{2}{ }^{2}$; eluent: 1:2 Pet. ether/Et $\left.2 \mathrm{O}\right) ;[\alpha]^{22}{ }_{\mathrm{D}}=$ $-248.4\left(c=0.42, \mathrm{CHCl}_{3}\right) ; v_{\max }($ film $) / \mathrm{cm}^{-1} 2922,2851(\mathrm{CH}), 1727\left(\mathrm{C}=\mathrm{O}_{\text {ester }}\right), 1679$ $\left(\mathrm{C}=\mathrm{O}_{\text {ketone }}\right) ;{ }^{1} \mathrm{H}$ NMR $\left(400 \mathrm{MHz}, \mathrm{CDCl}_{3}\right): \delta 6.82(1 \mathrm{H}, \mathrm{d}, J=4.0, \mathrm{H}-14), 5.71-5.63$ (1H, m, H-11), 5.41 (1H, dd, $J=9.0,4.5, \mathrm{H}-3), 4.86$ (1H, ddd, $J=16.0,15.0,1.0, \mathrm{H}-$ 10), 4.90-4.81 (1H, m, H-7), 3.37 (1H, d, $J=13.5, \mathrm{H}-5 \mathrm{a}), 3.30$ (1H, d, $J=13.5, \mathrm{H}-$ $5 \mathrm{~b}), 3.16(1 \mathrm{H}, \mathrm{ddd}, J=15.0,7.0,0.5, \mathrm{H}-12 \mathrm{a}), 2.68(1 \mathrm{H}, \mathrm{ddd}, J=17.5,10.0,5.5, \mathrm{H}-$ 1a), 2.52-2.41 (2H, m, H-12b and H-1b), 2.34-2.17 (4H, m, H-9a, 9b and H-2a, 2b), $1.25(3 \mathrm{H}, \mathrm{d}, J=6.0, \mathrm{H}-8) ;{ }^{13} \mathrm{C}$ NMR (100 MHz, $\left.\mathrm{CDCl}_{3}\right): \delta 197.8$ (C-15), $166.2(\mathrm{C}-4)$, 165.8 (C-6), 143.9 (C-13), 141.5 (C-14), 130.8 (C-11), 129.6 (C-10), 74.0 (C-7), 68.6 (C-3), 43.4 (C-5), 37.8 (C-9), 33.7 (C-1), 28.9 (C-12), 27.2 (C-2), 20.0 (C-8); m/z: 301 [MNa] ${ }^{+}$; HRMS (ESI): calcd. for $\mathrm{C}_{15} \mathrm{H}_{18} \mathrm{NaO}_{5}, 301.1046$. Found: [MNa] $]^{+}, 301.1039$ (2.3 ppm error). 
Minor isomer $(\mathrm{Z}), R_{f} 0.29\left(\mathrm{AgNO}_{3}\right.$ doped $\mathrm{SiO}_{2}$; eluent: $1: 2$ Pet. ether/Et $\left.2 \mathrm{O}\right) ;[\alpha]^{22}{ }_{\mathrm{D}}=$ -163.7 ( $\left.c=0.16, \mathrm{CHCl}_{3}\right) ; v_{\max }\left(\right.$ film) $/ \mathrm{cm}^{-1} 2921,2850(\mathrm{CH}), 1728\left(\mathrm{C}=\mathrm{O}_{\text {ester }}\right), 1680$ $\left(\mathrm{C}=\mathrm{O}_{\text {ketone }}\right) ;{ }^{1} \mathrm{H} \mathrm{NMR}\left(400 \mathrm{MHz}, \mathrm{CDCl}_{3}\right): \delta 6.84-6.80(1 \mathrm{H}, \mathrm{m}, \mathrm{H}-14), 5.78-5.62(2 \mathrm{H}$, m, H-10 and H-11), 5.55 (1H, ddd, $J=9.5,4.5,1.0, \mathrm{H}-3), 5.15-5.06$ (1H, m, H-7), 3.40 (1H, d, $J=16.0, \mathrm{H}-5 \mathrm{a}), 3.31$ (1H, d, $J=16.0, \mathrm{H}-5 \mathrm{~b}), 3.05$ (1H, dd, $J=16.5,7.0$, H-12a), 2.78 (1H, tdd, $J=16.5,8.0,1.5, \mathrm{H}-12 \mathrm{~b}), 2.67$ (1H, ddd, $J=16.5,9.5,5.0, \mathrm{H}-$ 1a), 2.47 (1H, ddd, $J=16.5,7.5,5.0, \mathrm{H}-1 \mathrm{~b}), 2.38-2.10$ (4H, m, H-9a, H-9b and H-2a, $\mathrm{H}-2 \mathrm{~b}), 1.35$ (3H, d, $J=6.5, \mathrm{H}-8) ;{ }^{13} \mathrm{C} \mathrm{NMR}\left(100 \mathrm{MHz}, \mathrm{CDCl}_{3}\right): \delta 198.7(\mathrm{C}-15), 166.9$ (C-4), 166.3 (C-6), 140.5 (C-13), 139.7 (C-14), 128.2 (C-10), 127.6 (C-11), 72.3 (C7), 67.9 (C-3), 42.4 (C-5), 34.3 (C-1), 32.8 (C-9), 27.1 (C-2), 26.1 (C-12), 18.5 (C-8); $m / z$ (ESI): 301 [MNa] ${ }^{+}$; HRMS (ESI): calcd. for $\mathrm{C}_{15} \mathrm{H}_{18} \mathrm{NaO}_{5}$, 301.1046. Found: $[\mathrm{MNa}]^{+}, 301.1054$ (2.42 ppm error).

\section{(2a $\left.S, 2 \mathrm{a}^{1} S, 10 S\right)-10-M e t h y l-3,4,5 \mathrm{a}, 6,9,10$-hexahydrobenzofuro[3,4-cd] oxecine-}

\section{1,5,12(2a $\left.H, 2 \mathrm{a}^{1} H, 12 \mathrm{a} H\right)$-trione (22b)}

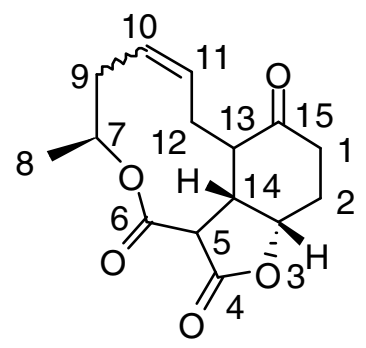

A solution of enone 22 ( $0.043 \mathrm{~g}, 0.15 \mathrm{mmol}, 1.0$ eq.) in dry THF (5 mL) was added to a stirred solution of $\mathrm{NaH}[60 \% \mathrm{w} / \mathrm{w}(0.007 \mathrm{~g}, 0.19 \mathrm{mmol}, 1.2 \mathrm{eq})$.$] in dry THF (5$ $\mathrm{mL}$ ) at $0{ }^{\circ} \mathrm{C}$. After $10 \mathrm{~min}$, the reaction was warmed to $\mathrm{rt}$ and stirred for $1 \mathrm{~h}$, when the reaction was judged to be complete by TLC analysis. The reaction was cooled to $0^{\circ} \mathrm{C}$, quenched carefully with sat. $\mathrm{NH}_{4} \mathrm{Cl}$ (aq.) solution $(1 \mathrm{~mL})$, diluted with EtOAc (20 $\mathrm{mL}$ ), $\mathrm{Na}_{2} \mathrm{SO}_{4}$ added (5 g) and the resulting suspension stirred vigorously for $10 \mathrm{~min}$. The suspension was filtered through a plug of $\mathrm{SiO}_{2}$, the plug washed with EtOAc (30 $\mathrm{mL}$ ) and the filtrate concentrated in vacuo. The resulting crude product was purified by flash column chromatography on silica gel (eluent: $2: 3$ Pet. ether/EtOAc) to give the title compound $22 \boldsymbol{b}(0.036 \mathrm{~g}, 84 \%)$ as a white amorphous solid, $R_{f} 0.31$ (1:1 Pet. 
ether/EtOAc); $[\alpha]^{23}=-56.7\left(c=0.34, \mathrm{CHCl}_{3}\right) ; v_{\max }($ film $) / \mathrm{cm}^{-1} 3056\left(\mathrm{CH}_{\text {alkene }}\right)$, 2972, $2933(\mathrm{CH}), 1777\left(\mathrm{C}=\mathrm{O}_{\text {ester }}\right), 1717\left(\mathrm{C}=\mathrm{O}_{\text {ketone }}\right) ;{ }^{1} \mathrm{H}$ NMR (400 MHz, $\left.\mathrm{CDCl}_{3}\right): \delta$ 5.52-5.39 (1H, m, H-10, H-10 $)$, 5.28-5.18 (1H, m H-11 $\left.{ }_{E}, \mathrm{H}-11_{Z}\right), 5.10-4.98(1 \mathrm{H}, \mathrm{m}$, $\left.\mathrm{H}-7_{E}, \mathrm{H}-7_{Z}\right), 4.69-4.57\left(1 \mathrm{H}, 2 \mathrm{x}\right.$ ddd, $\left.J=12.5,8.5,4.0, \mathrm{H}-3_{E}, \mathrm{H}-3_{Z}\right), 3.86(1 \mathrm{H}, \mathrm{d}, J=$ $\left.10.0, \mathrm{H}-5_{Z}\right), 3.55\left(1 \mathrm{H}, \mathrm{d}, J=11.5, \mathrm{H}-5_{E}\right), 3.15-3.08(1 \mathrm{H}, \mathrm{ddd}, J=11.0,10.0,4.5, \mathrm{H}-$ 14z), 2.98-2.92 (1H, m, H-14 E), 2.90-1.82 (9H, m, H-9, H-12, H-13, H-1, H-2), 1.37 $\left(3 \mathrm{H}, \mathrm{d}, J=6.5, \mathrm{H}-8_{\mathrm{Z}}\right), 1.31\left(3 \mathrm{H}, \mathrm{d}, J=6.5, \mathrm{H}-8_{E}\right) ;{ }^{13} \mathrm{C} \mathrm{NMR}\left(100 \mathrm{MHz}, \mathrm{CDCl}_{3}\right)$, selected signals for major isomer $\delta 209.5$ (C-15), 172.4 (C-4), 168.0 (C-6), 131.5 (C10), 129.2 (C-11), 77.9 (C-3), 73.2 (C-7), 55.6 (C-5), 50.6 (C-14), 43.8 (C-13), 35.5, 35.0, 33.6, 25.0, (C-9, C-12, C-1, C-2), 19.6 (C-8); m/z (ESI): $301[\mathrm{MNa}]^{+}$; HRMS: HRMS (ESI): calcd. for $\mathrm{C}_{15} \mathrm{H}_{18} \mathrm{NaO}_{5}, 301.1046$. Found: [MNa] $]^{+}, 301.1054$ (2.47 ppm error).

(2aS,2a $1 S, 5 \mathrm{a} R, 10 S, 12 \mathrm{a} S)-10-$ Methyloctahydrobenzofuro[3,4-cd] oxecine-

\section{1,5,12(2aH,2a1H,12aH)-trione (23) [+(24)]}
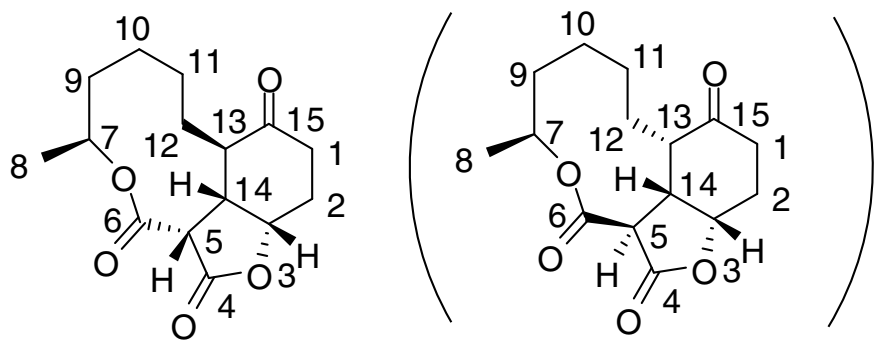

To a stirred solution of alkene 22b (0.059 g, 0.21 mmol, 1.0 eq.) in dry THF (5 mL), was added $\mathrm{Pd} / \mathrm{C}[3 \% \mathrm{w} / \mathrm{w}(0.075 \mathrm{~g}, 0.021 \mathrm{mmol}, 0.10 \mathrm{eq})$.$] and the system purged via$ $5 \mathrm{x}$ vacuum/argon cycles followed by $5 \mathrm{x}$ vacuum/hydrogen cycles. The system was maintained under a hydrogen atmosphere and the reaction stirred at $\mathrm{rt}$ for $20 \mathrm{~h}$, when judged to be complete by TLC analysis. The reaction mixture was diluted with $\mathrm{Et}_{2} \mathrm{O}$ $(5 \mathrm{~mL})$, filtered through a pad of Celite ${ }^{\circledR}$ and the pad washed successively with $\mathrm{Et}_{2} \mathrm{O}$ $(20 \mathrm{~mL})$, EtOAc $(20 \mathrm{~mL})$ and $\mathrm{MeOH}(20 \mathrm{~mL})$. The filtrate was concentrated in vacuo and the resulting crude product was purified by flash column chromatography on silica gel (eluent: $\left.100 \% \mathrm{Et}_{2} \mathrm{O}\right)$ to give the title compound $23(+24)(0.055 \mathrm{~g}, 93 \%)$ as an amorphous white solid in a 2:1 ration of diastereomers. For major diastereomer 23, $R_{f} 0.32\left(100 \% \mathrm{Et}_{2} \mathrm{O}\right) ;[\alpha]_{\mathrm{D}}^{22}=-10.8\left(c=0.41, \mathrm{CHCl}_{3}\right) ; v_{\max }(\mathrm{film}) / \mathrm{cm}^{-1} 2918,2850$ 
(CH), $1771\left(\mathrm{C}=\mathrm{O}_{\text {ester }}\right), 1715\left(\mathrm{C}=\mathrm{O}_{\text {ketone }}\right) ;{ }^{1} \mathrm{H}$ NMR (400 MHz, $\left.\mathrm{CDCl}_{3}\right): \delta 5.21(1 \mathrm{H}$, dqd, $J=11.0,6.5,2.0, \mathrm{H}-7), 4.61$ (1H, ddd, $J=12.0,9.0,4.5, \mathrm{H}-3), 3.88$ (1H, d, $J=$ 11.5, H-5), 2.88 (1H, ddd, $J=12.0,11.5,9.0, \mathrm{H}-14), 2.63-2.50$ (2H, m, H-13, H-1a), 2.46-2.35 (1H, m, H-2a), 2.29-2.22 (2H, m, H-1b, H-2b), 1.96 (1H, ddd, J = 7.5, 7.0, 4.0, H-12a), 1.87-1.82 (1H, m, H-11a), 1.76-1.69 (1H, m, H-10a), 1.49-1.30 (5H, m, H-9a, H-9b, H-10b, H-11b, H-12b), 1.26 (3H, d, $J=6.5, \mathrm{H}-8) ;{ }^{13} \mathrm{C}$ NMR (100 MHz, $\left.\mathrm{CDCl}_{3}\right): \delta 210.4(\mathrm{C}-15), 172.1$ (C-6), 168.3 (C-4), 77.9 (C-7), 75.4 (C-3), $51.0(\mathrm{C}-5)$, 44.3 (C-13), 40.4 (C-14), 35.3 (C-1), 35.1 (C-11), 26.7 (C-2), 26.0 (C-12), 24.3 (C-9), 21.9 (C-10), 20.9 (C-8); m/z (ESI): $298 \quad\left[\mathrm{MNH}_{4}\right]^{+}$; HRMS (ESI): calcd. for $\mathrm{C}_{15} \mathrm{H}_{24} \mathrm{NO}_{5}$, 298.1649. Found: $\left[\mathrm{MNH}_{4}\right]^{+}$, 298.1651 (0.7 ppm error). For minor diastereomer 24, $R_{f} 0.27\left(100 \% \mathrm{Et}_{2} \mathrm{O}\right) ; v_{\max }(\mathrm{film}) / \mathrm{cm}^{-1} 2920,2853(\mathrm{CH}), 1769$ $\left(\mathrm{C}=\mathrm{O}_{\text {ester}}\right), 1712\left(\mathrm{C}=\mathrm{O}_{\text {ketone}}\right) ;{ }^{1} \mathrm{H}$ NMR $\left(400 \mathrm{MHz}, \mathrm{CDCl}_{3}\right): \delta 5.25-5.18(1 \mathrm{H}, \mathrm{m}, \mathrm{H}-7)$, 5.03-4.98 (1H, m, H-3), 3.99-3.93 (1H, m, H-14), 3.26 (1H, d, J = 13.5, H-5), 2.56 (1H, ddd, $J=12.0,6.0,2.0, \mathrm{H}-13), 2.49-2.41$ (2H, m, H-1a, H-2a), 2.38.2.33 (1H, m, H-1b), 2.13-1.82 (4H, m, H-2b, H-10a, H-11a, H-12a), 1.59-1.36 (5H, m, H-9a, H-9b, $\mathrm{H}-10 \mathrm{~b}, \mathrm{H}-11 \mathrm{~b}, \mathrm{H}-12 \mathrm{~b}), 1.30(3 \mathrm{H}, \mathrm{d}, J=6.5, \mathrm{H}-8) .{ }^{13} \mathrm{C} \mathrm{NMR}\left(100 \mathrm{MHz}, \mathrm{CDCl}_{3}\right): \delta$ 209.6 (C-15), 170.5 (C-6), 165.3 (C-4), 75.9 (C-7), 75.1 (C-3), 49.7 (C-5), 48.2 (C13), 42.7 (C-14), 36.5 (C-1), 30.7 (C-11), 30.0 (C-2), 28.9 (C-12), 23.3 (C-9), 23.1 (C-10), 17.5 (C-8).

\section{$(2 E, 4 E, 6 E)$-Ethyl 7-(tributylstannyl)hepta-2,4,6-trienoate (26)}

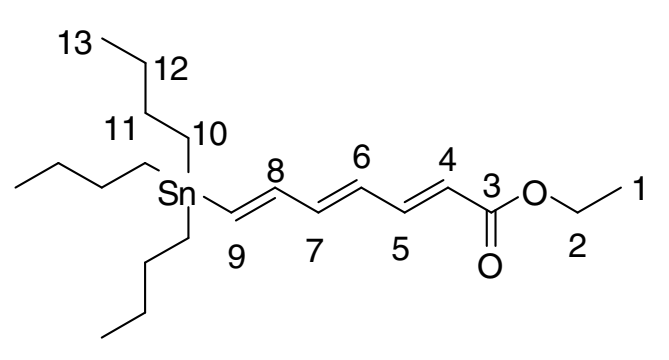

$\operatorname{Pd}(\mathrm{dba})_{2}(0.067 \mathrm{~g}, 0.12 \mathrm{mmol}, 0.08$ eq. $)$ was added to stirred solution of $(E)-1,2-$ bis(tributylstannyl)ethene $(0.89 \mathrm{~mL}, 1.68 \mathrm{mmol}, 1.15$ eq. $)$ and $(2 E, 4 E)$-ethyl 5bromopenta-2,4-dienoate ( $0.300 \mathrm{~g}, 1.46 \mathrm{mmol}, 1.0$ eq.) in dry, argon sparged THF (25 $\mathrm{mL}$ ). The resulting brown/black solution was purged via $10 \mathrm{x}$ vacuum/argon cycles and maintained under an argon atmosphere. The reaction mixture was heated to reflux $\left(67^{\circ} \mathrm{C}\right)$ and stirred for $1.5 \mathrm{~h}$, when the reaction was judged to be complete by TLC 
analysis. After allowing to cool to rt, the solvent was removed in vacuo, the crude residue dissolved in $\mathrm{Et}_{2} \mathrm{O}$ and filtered through a plug of $\mathrm{SiO}_{2}$. The filtrate was concentrated in vacuo and the resulting crude product was purified by flash column chromatography on silica gel (eluent: 19:1 Pet. ether/EtOAc) to give the title compound 26 (0.357 g, $55 \%)$ as a yellow oil, $R_{f} 0.44$ (9:1 Pet. ether/EtOAc); $v_{\max }$ (film)/cm ${ }^{-1}$ 2957, $2926(\mathrm{CH}), 1714(\mathrm{C}=\mathrm{O}), 1623(\mathrm{C}=\mathrm{C}) ;{ }^{1} \mathrm{H}$ NMR (400 MHz, $\left.\mathrm{CDCl}_{3}\right)$ : $\delta 7.31$ (1H, dd, $J=15.5,11.5, \mathrm{H}-5), 6.69-6.44$ (3H, m, H-7, H-8, H-9), 6.25 (1H, dd, $J=14.5,11.5, \mathrm{H}-6), 5.88$ (1H, d, $J=15.5, \mathrm{H}-4), 4.21$ (2H, q, $J=7.0, \mathrm{H}-2), 1.68-1.44$ (6H, m, H-12), 1.42-1.25 (9H, H-1 and H-11), 0.95-0.84 (15H, m, H-10 and H-13). ${ }^{13} \mathrm{C} \mathrm{NMR}\left(100 \mathrm{MHz}, \mathrm{CDCl}_{3}\right): \delta 167.6(\mathrm{C}-3), 146.4(\mathrm{C}-5), 145.3$ (C-alkene), $143.4(\mathrm{C}$ alkene), 142.7 (C-alkene), 129.2 (C-6), 121.8 (C-4), 60.8 (C-2), 29.4 (C-Bu), 28.8 (C$\mathrm{Bu}), 27.8(\mathrm{C}-\mathrm{Bu}), 27.4(\mathrm{C}-\mathrm{Bu}), 17.9(\mathrm{C}-\mathrm{Bu}), 14.9(\mathrm{C}-\mathrm{Bu}), 14.3(\mathrm{C}-\mathrm{Bu}), 14.2(\mathrm{C}-\mathrm{Bu})$, 10.2, (C-Bu); m/z (CI) $443\left(\mathrm{C}_{21} \mathrm{H}_{39} \mathrm{O}_{2}{ }^{120} \mathrm{Sn}, 48\right), 308$ (100); HRMS(CI): calcd. for $\mathrm{C}_{21} \mathrm{H}_{39} \mathrm{O}_{2}{ }^{116} \mathrm{Sn}, 439.1976$. Found: $\mathrm{MH}^{+}, 439.1962$ (1.3 ppm error).

\section{$(2 E, 4 E, 6 E)$-Ethyl-7-((2a $S, 2 \mathrm{a} 1 S, 10 S)$-10-methyl-5,12-dioxo-}

\section{2a,2a1,3,4,5,5a,6,7,8,9,10,12-dodecahydrobenzofuro[3,4-cd]oxecin-1-yl)hepta-}

\section{2,4,6-trienoate (27)}

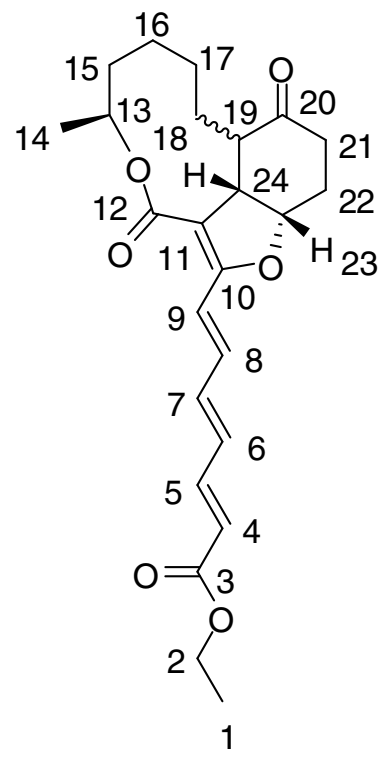

a) Triflate formation

To a stirred solution of substrate 23 (0.033 g, $0.12 \mathrm{mmol}, 1.0$ eq.) in dry, argon sparged DME $(4.0 \mathrm{~mL})$ at $-40{ }^{\circ} \mathrm{C}$, was added KHMDS [0.97 $\mathrm{M}$ in DME $(0.15 \mathrm{~mL}$, $0.14 \mathrm{mmol}, 1.2$ eq.)]. After $25 \mathrm{~min}$, triflic anhydride (0.024 mL, $0.14 \mathrm{mmol}, 1.2$ eq.) 
was added and the reaction stirred at $-40{ }^{\circ} \mathrm{C}$ for $20 \mathrm{~min}$, warmed to $\mathrm{rt}$ and stirred for 1 h. The reaction mixture was cooled to $0{ }^{\circ} \mathrm{C}$, quenched carefully with sat. $\mathrm{NH}_{4} \mathrm{Cl}$ (aq.) solution $(2 \mathrm{~mL})$ and the aqueous phase extracted with EtOAc $(4 \times 6 \mathrm{~mL})$. The combined organic phases were dried $\left(\mathrm{Na}_{2} \mathrm{SO}_{4}\right)$ and concentrated in vacuo to give the crude triflate $\mathbf{2 5}$ as a green/black gummy solid, which was carried forward to the next step without further purification.

\section{b) Stille coupling with triene $\mathbf{2 6}$}

A flask containing $\mathrm{LiCl}(0.015 \mathrm{~g}, 0.35 \mathrm{mmol}, 3.0 \mathrm{eq}$.$) was heated with the heat-gun$ for $5 \mathrm{~min}$ under high-vacuum. After cooling to rt, $\mathrm{Pd}_{2}(\mathrm{dba})_{3}(0.011 \mathrm{~g}, 0.012 \mathrm{mmol}, 0.1$ eq.) and (2-furyl $)_{3} \mathrm{P}(0.008 \mathrm{~g}, 0.035 \mathrm{mmol}, 0.3$ eq.) were added and the system purged via $5 \mathrm{x}$ vacuum/argon cycles. A solution of the crude triflate 25 in dry, argon sparged DME (4.0 mL) followed by a solution of triene $26(0.091 \mathrm{~g}, 0.21 \mathrm{mmol}, 1.20$ eq.) in dry, argon sparged DME (4.0 mL) were added, the system was purged via $10 \mathrm{x}$ vacuum/argon cycles, maintained under an argon atmosphere, heated to reflux and allowed to stir for $18 \mathrm{~h}$, when the reaction was judged to be complete by TLC analysis. The reaction was allowed to cool to rt, filtered through a short pad of Celite ${ }^{\circledR}$ - $10 \% \mathrm{KF} /$ silica gel and concentrated in vacuo. The resulting crude product was purified by flash column chromatography on $10 \% \mathrm{KF} /$ silica gel (eluent: 3:2 Pet. ether/EtOAc)) to give the title compound $27(0.027 \mathrm{~g}, 54 \%)$ as an amorphous brightyellow solid in a $3: 2$ ratio of diastereomers, $R_{f} 0.50_{\text {minor }}, 0.38_{\text {major }}$ (3:2 Pet. ether/EtOAc); $[\alpha]^{22}{ }_{\mathrm{D}}=+30.1\left(c=0.40, \mathrm{CHCl}_{3}\right) ; v_{\max }(\mathrm{film}) / \mathrm{cm}^{-1} 2928,2821(\mathrm{CH})$, $1712\left(\mathrm{C}=\mathrm{O}_{\text {ketone }}\right), 1631\left(\mathrm{C}=\mathrm{O}_{\alpha, \beta}\right.$ unsat. ester $) ;{ }^{1} \mathrm{H} \mathrm{NMR}\left(400 \mathrm{MHz}, \mathrm{CDCl}_{3}\right): \delta$ 7.36-7.27 $\left(2 \mathrm{H}, \mathrm{m}, \mathrm{H}-5_{\text {major }}, \mathrm{H}-5_{\text {minor }}\right), 7.19$ (1H, d, $\left.J=15.5, \mathrm{H}-9_{\text {major }}\right), 7.05(1 \mathrm{H}, \mathrm{d}, J=15.5, \mathrm{H}-$ $\left.9_{\text {minor }}\right), 6.93\left(1 \mathrm{H}, \mathrm{dd}, J=15.5,11.0, \mathrm{H}-8_{\text {major }}\right), 6.82$ (1H, dd, $\left.J=15.5,11.0, \mathrm{H}-8_{\text {minor }}\right)$, 6.72-6.57 (2H, m, H-7 major $_{\text {, H-7 }}$ minor $)$, 6.54-6.37 (2H, m, H-6 major $_{\text {, H-6 }}$ minor), 5.96 (1H, d, $\left.J=15.0, \mathrm{H}-4_{\text {minor }}\right), 5.89\left(1 \mathrm{H}, \mathrm{d}, J=15.0, \mathrm{H}-4_{\text {major }}\right), 5.14(1 \mathrm{H}, \mathrm{dd}, J=10.0,2.0, \mathrm{H}-$ $23_{\text {major }}$ ), 5.04-4.92 (3H, m, H-13 major $_{\text {, H-13 }}$ minor, H-23 $3_{\text {minor }}$ ), 4.20 (4H, 2 x q, J= 7.0, H$\left.2_{\text {major, }} \mathrm{H}-2_{\text {minor }}\right), 4.04$ (1H, dd, $\left.J=10.0,7.5, \mathrm{H}-24_{\text {major }}\right), 3.44-3.38$ (1H, m, H-24 $4_{\text {minor }}$ ),

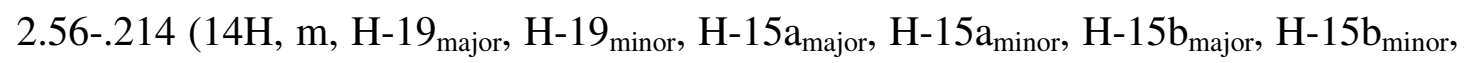
H-21 $\mathrm{a}_{\text {major }}$, H-21 $\mathrm{a}_{\text {minor }}, \mathrm{H}-21 \mathrm{~b}_{\text {major }}, \mathrm{H}-21 \mathrm{~b}_{\text {minor }}, \mathrm{H}-22 \mathrm{a}_{\text {major }}, \mathrm{H}-22 \mathrm{a}_{\text {minor }}, \mathrm{H}-22 \mathrm{~b}_{\text {major }}, \mathrm{H}-$ 22 $2_{\text {minor }}$ ), 1.97-1.70 (6H, m, H-16 $\mathrm{a}_{\text {major }}$, H-16 $\mathrm{a}_{\text {minor }}, \mathrm{H}-17 \mathrm{a}_{\text {major }}, \mathrm{H}-17 \mathrm{a}_{\text {minor }}, \mathrm{H}-18 \mathrm{a}_{\text {major }}$, H-18 $\mathrm{a}_{\text {minor }}$ ), 1.31-1.27 (12H, m, H-1 major $\left., \mathrm{H}-1_{\text {minor }}, \mathrm{H}-14_{\text {major }}, \mathrm{H}-14_{\text {minor }}\right), 1.12-0.78$ (6H,

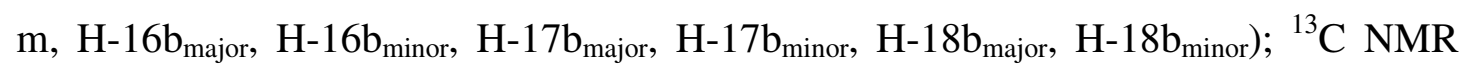


$\left(100 \mathrm{MHz}, \mathrm{CDCl}_{3}\right)$ selected signals for major diastereomer: $\delta 213.0(\mathrm{C}-20), 167.8(\mathrm{C}-$ 10), 165.9 (C-12), 164.6 (C-3), 144.4 (C-5), 140.0 (C-7), 136.6 (C-8), 135.4 (C-6), 123.9 (C-4), 122.9 (C-9), 108.5 (C-11), 83.8 (C-13), 81.3 (C-23), 61.0 (C-2), 49.7 (C19), 44.2 (C-24), 35.4 (C-21), 34.0 (C-22), 32.3 (C-15), 30.0 (C-14), 24.7 (C-16), 24.1 (C-17), 23.9 (C-18), 14.5 (C-1); m/z (ESI): 415 [MH']; HRMS: calcd. for $\mathrm{C}_{24} \mathrm{H}_{31} \mathrm{O}_{6}$, 415.2115. Found: $\left[\mathrm{MH}^{+}\right], 415.2125$ (2.28 ppm error).

(1) Barros, M. T.; Maycock, C. D.; Ventura, M. R. J. Chem. Soc., Perkin Trans. 1 2001, 166-173.

(2) Ruprah, P. K.; Cros, J.-P.; Pease, J. E.; Whittingham, W. G.; Williams, J. M. J. Eur. J. Org. Chem. 2002, 3145-3152. 

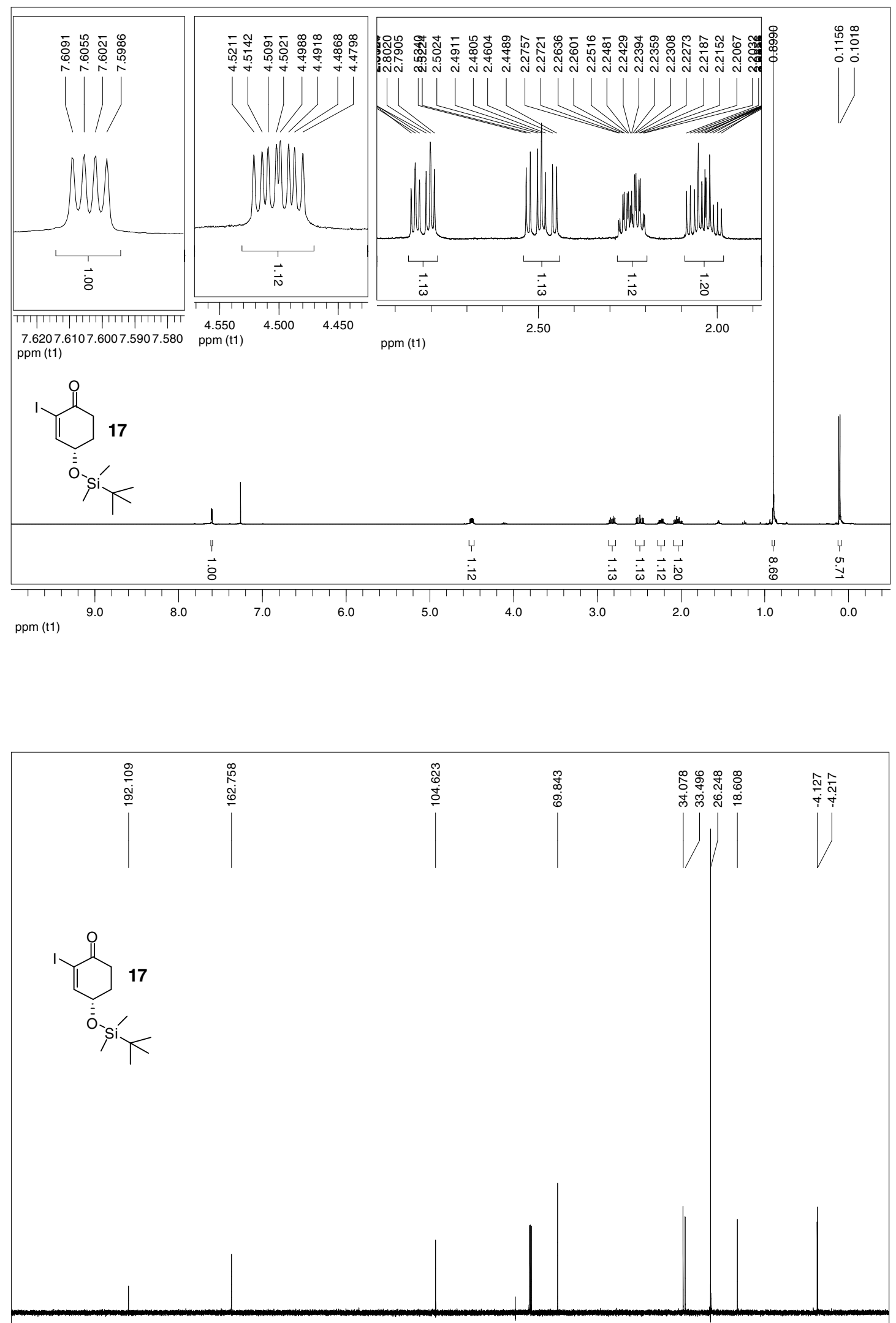

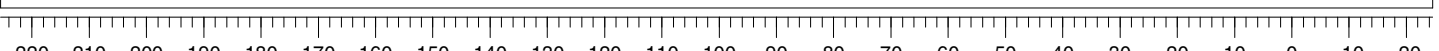
$\begin{array}{lllllllllllllllllllllllll}220 & 210 & 200 & 190 & 180 & 170 & 160 & 150 & 140 & 130 & 120 & 110 & 100 & 90 & 80 & 70 & 60 & 50 & 40 & 30 & 20 & 10 & 0 & -10 & -20\end{array}$ ppm (t1) 

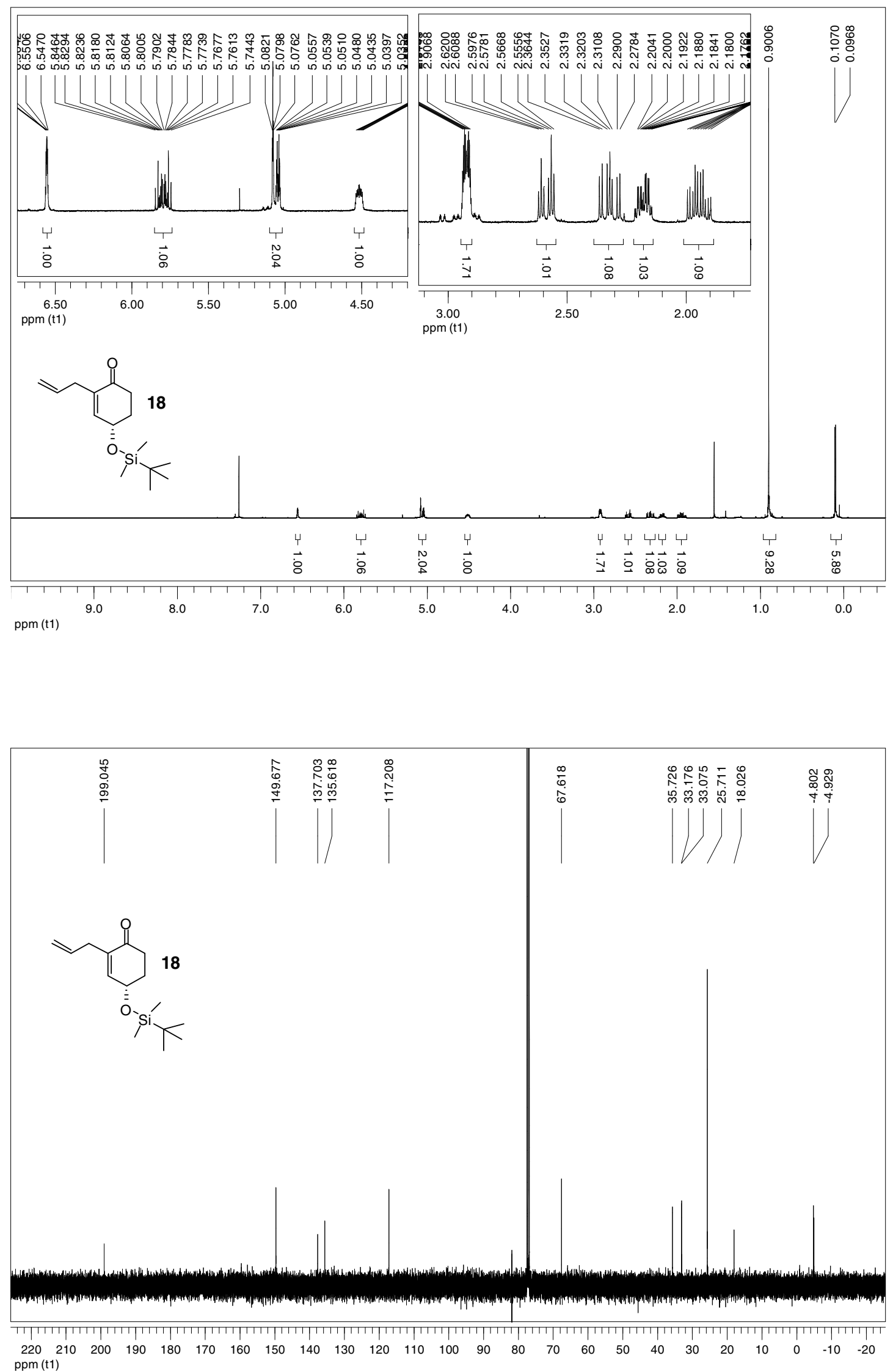

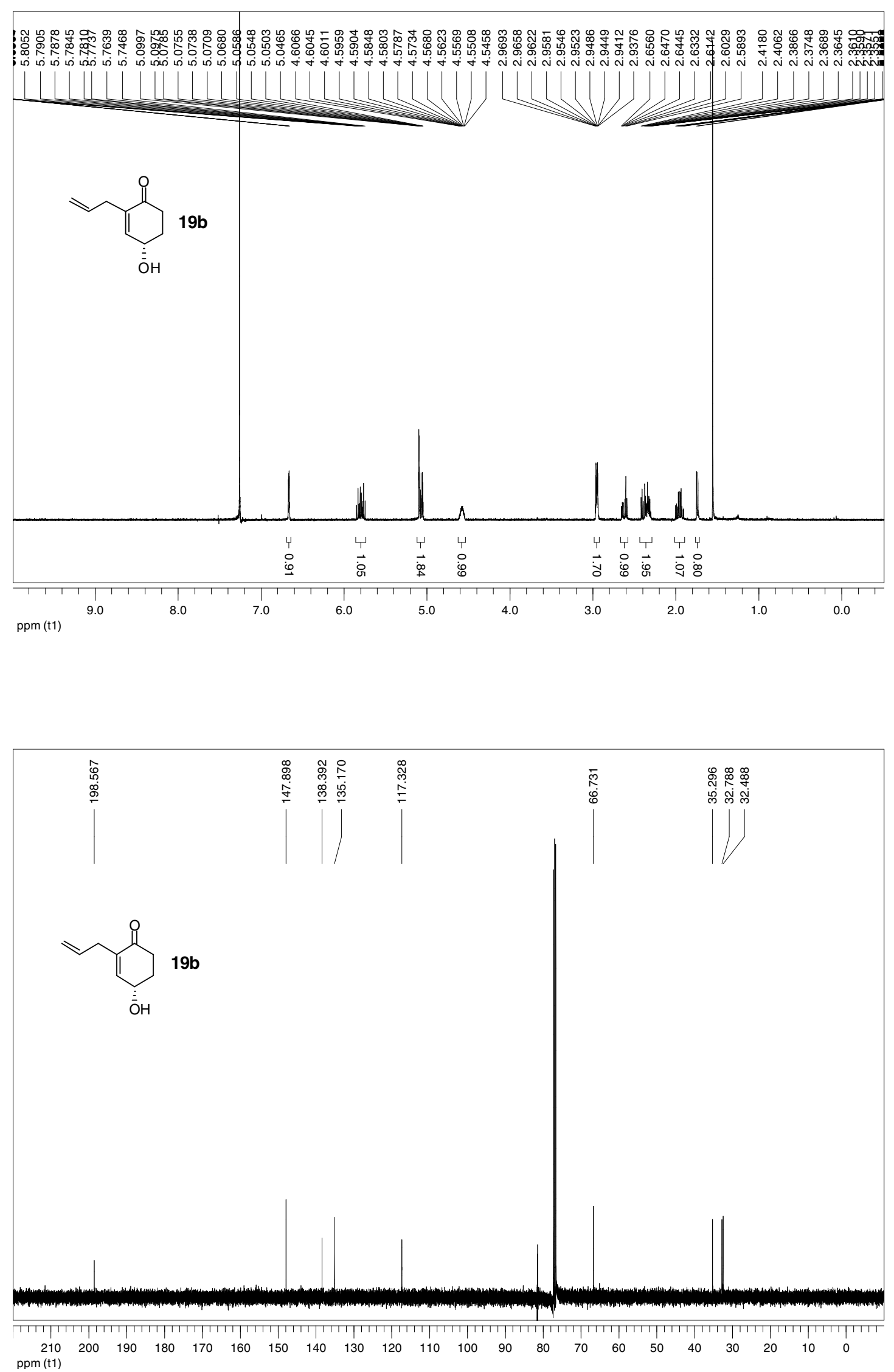

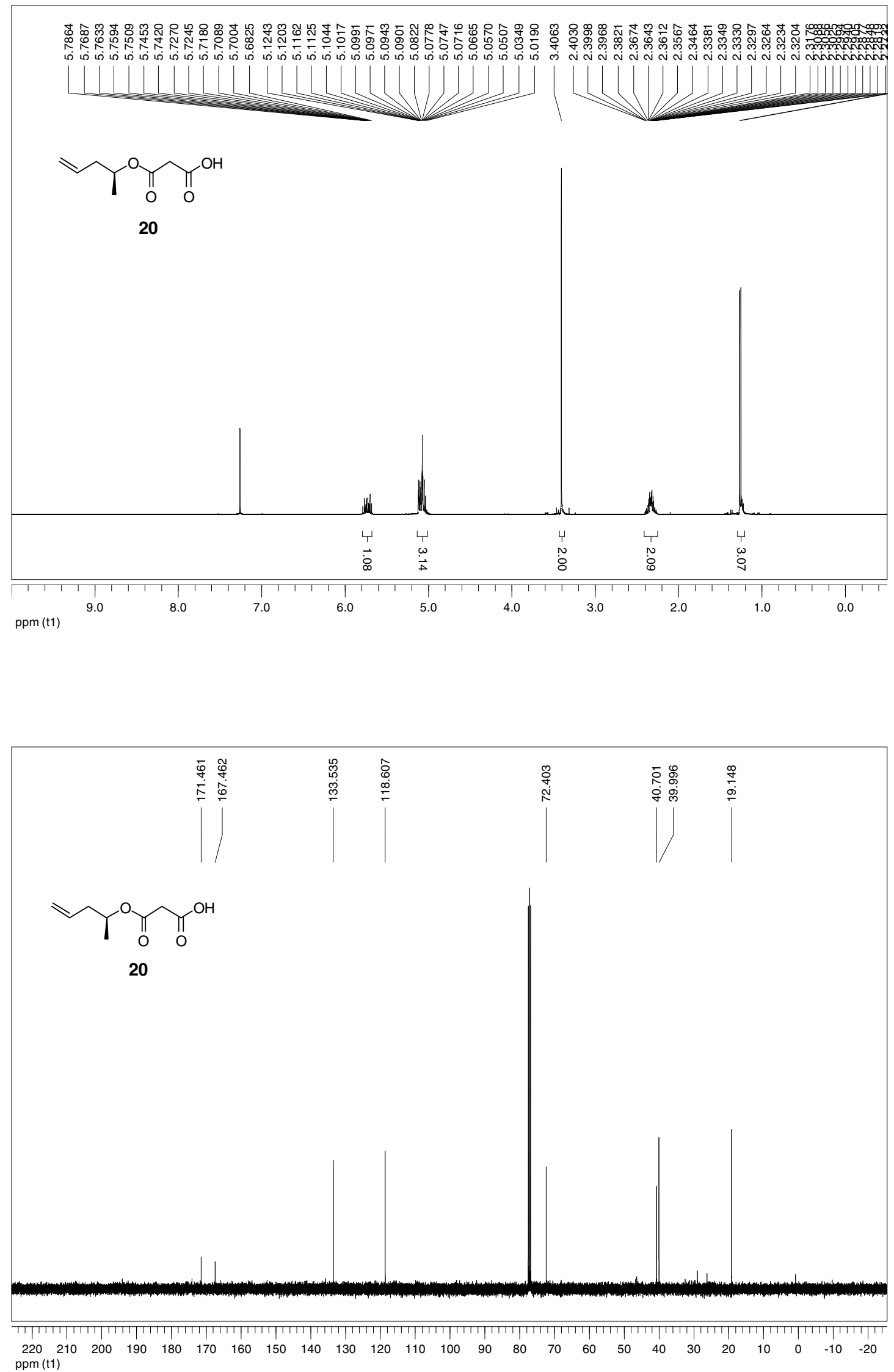


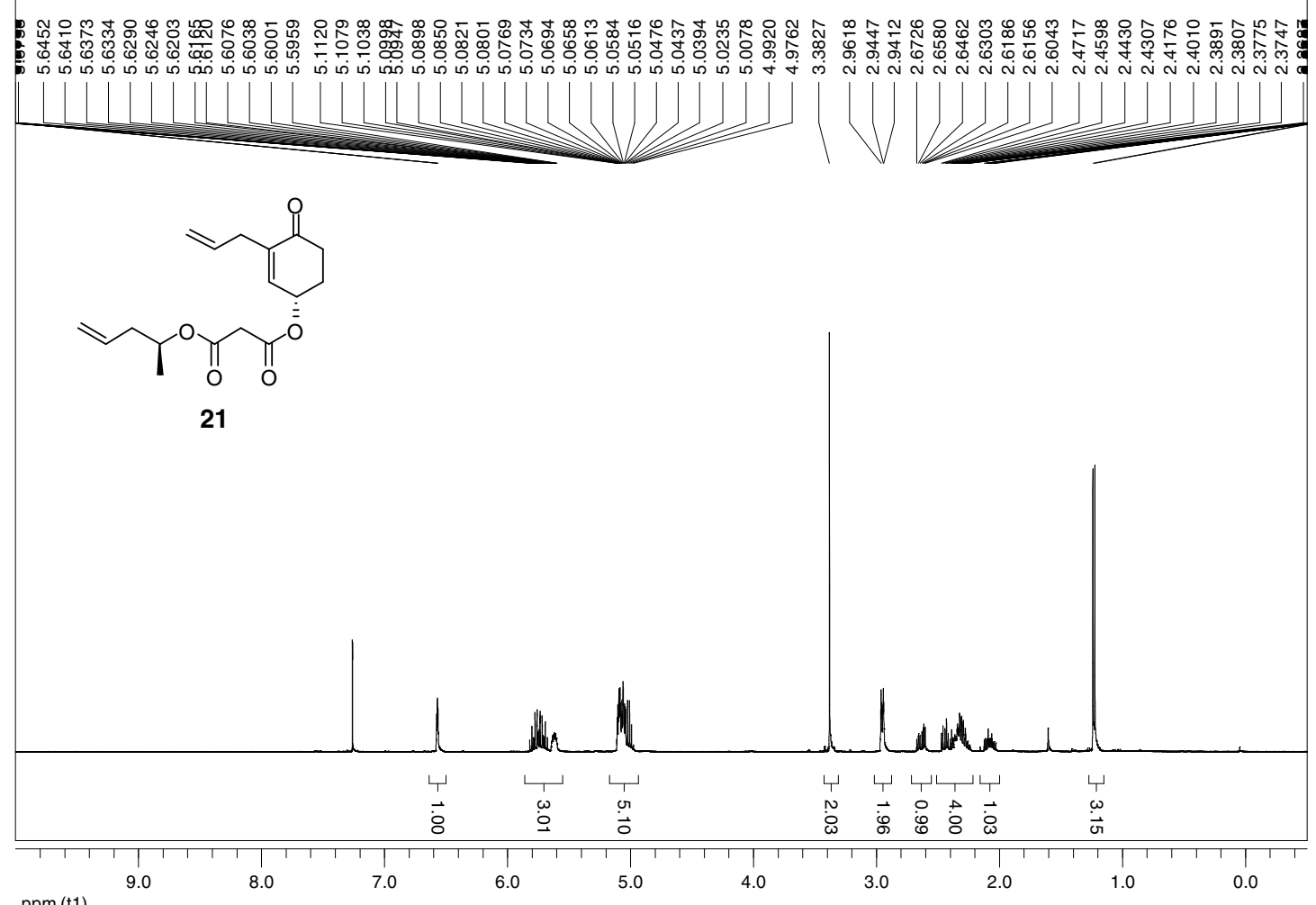

ppm (t1)

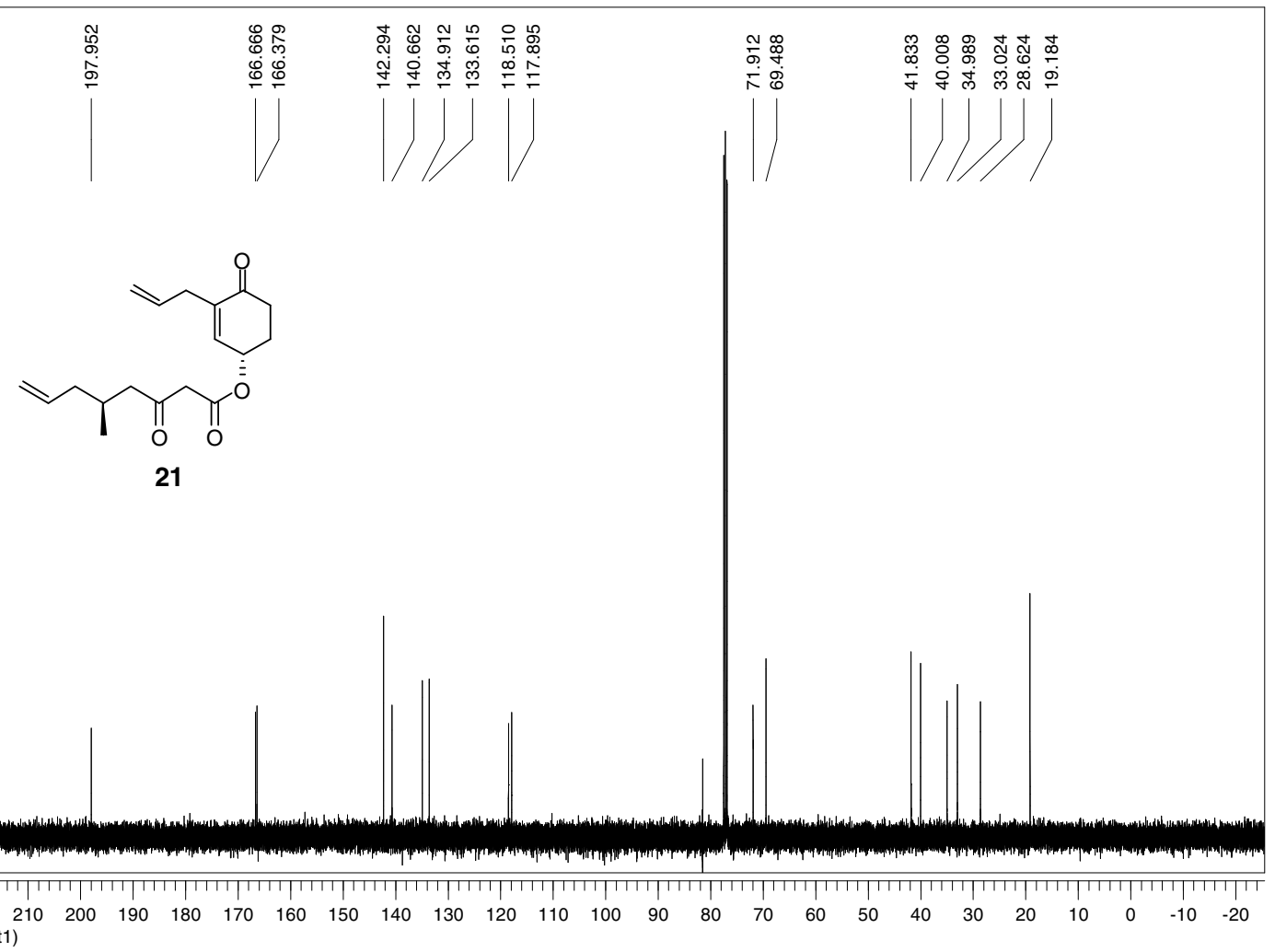



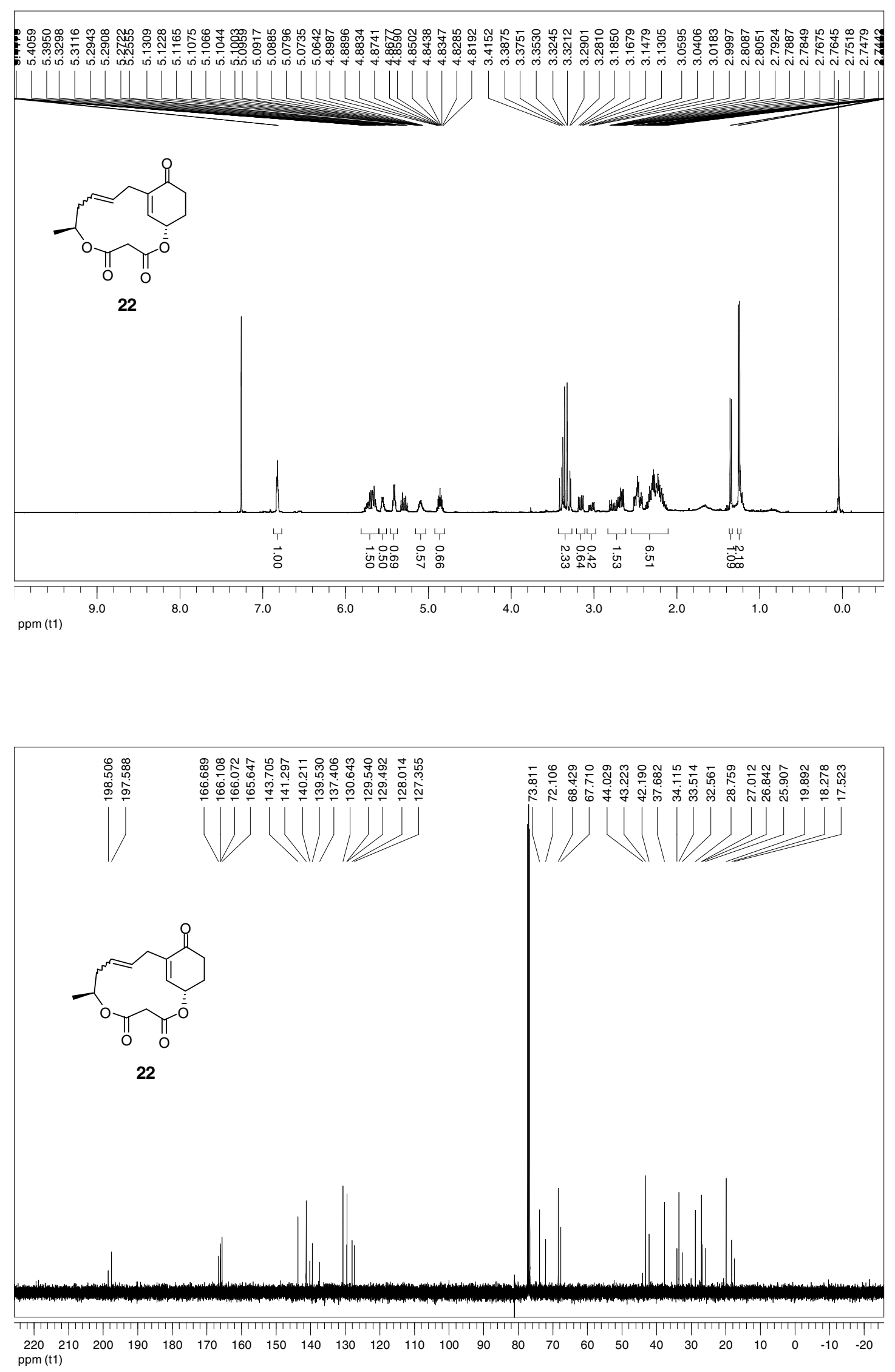

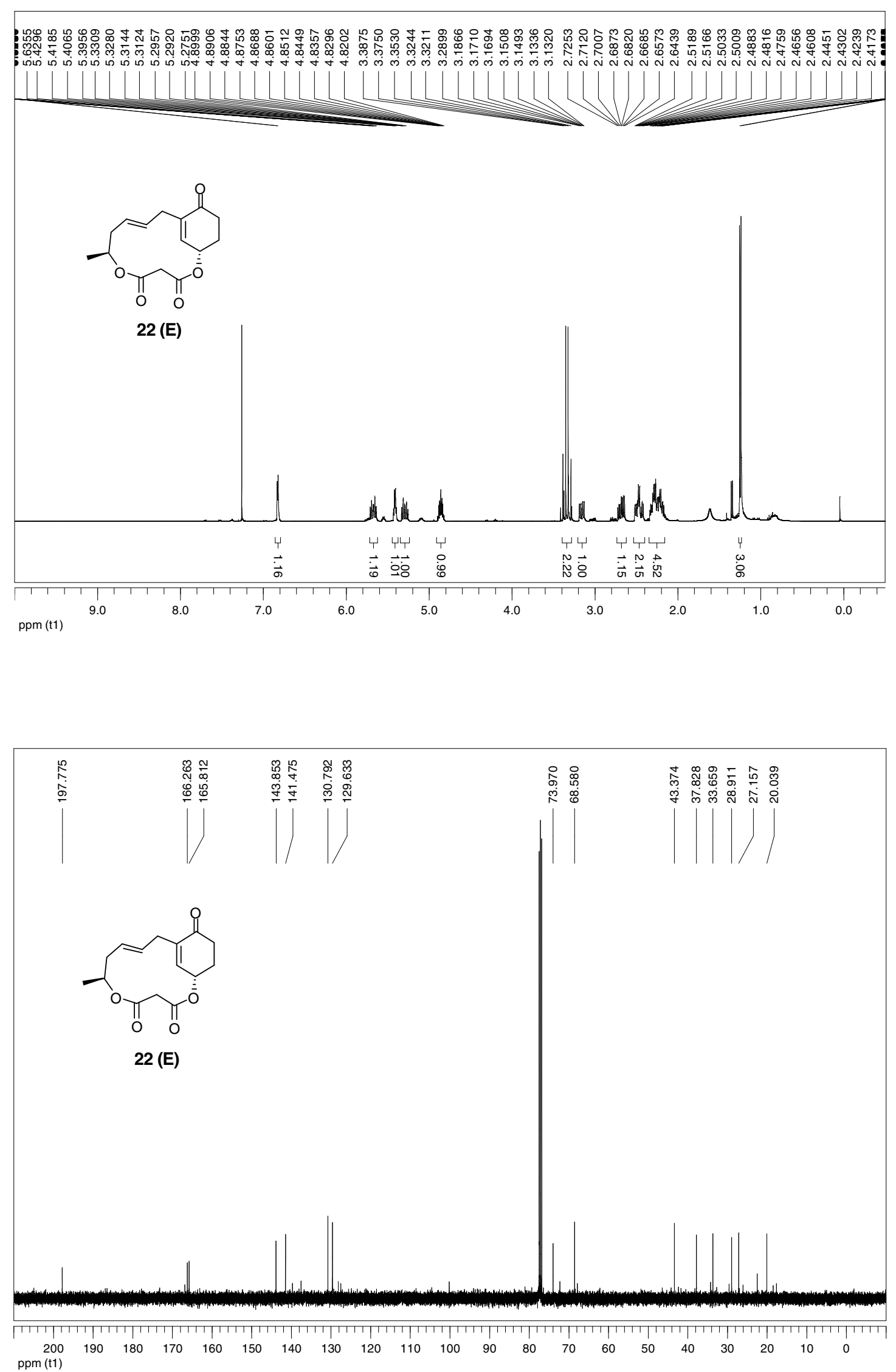


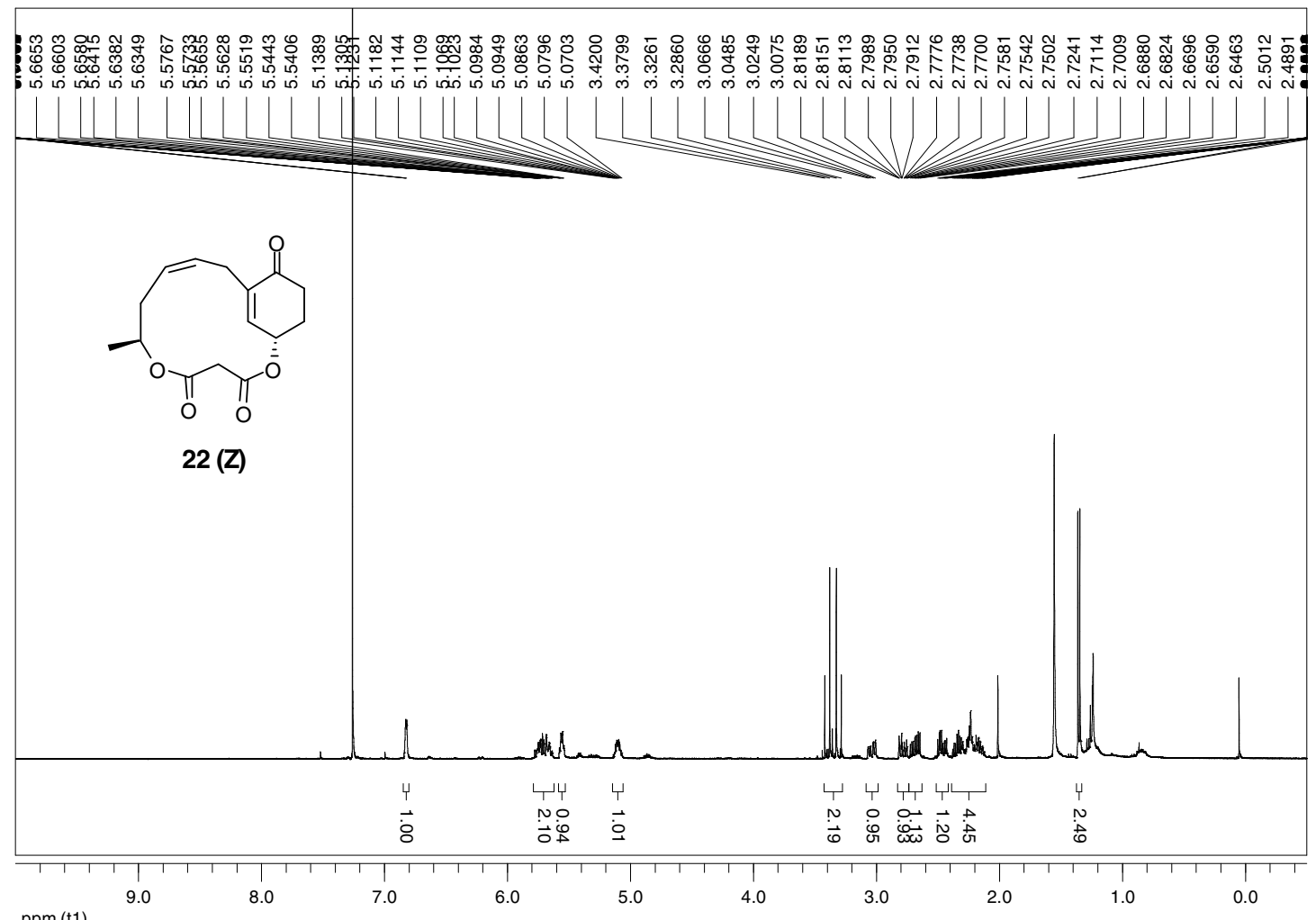

ppm (t1)

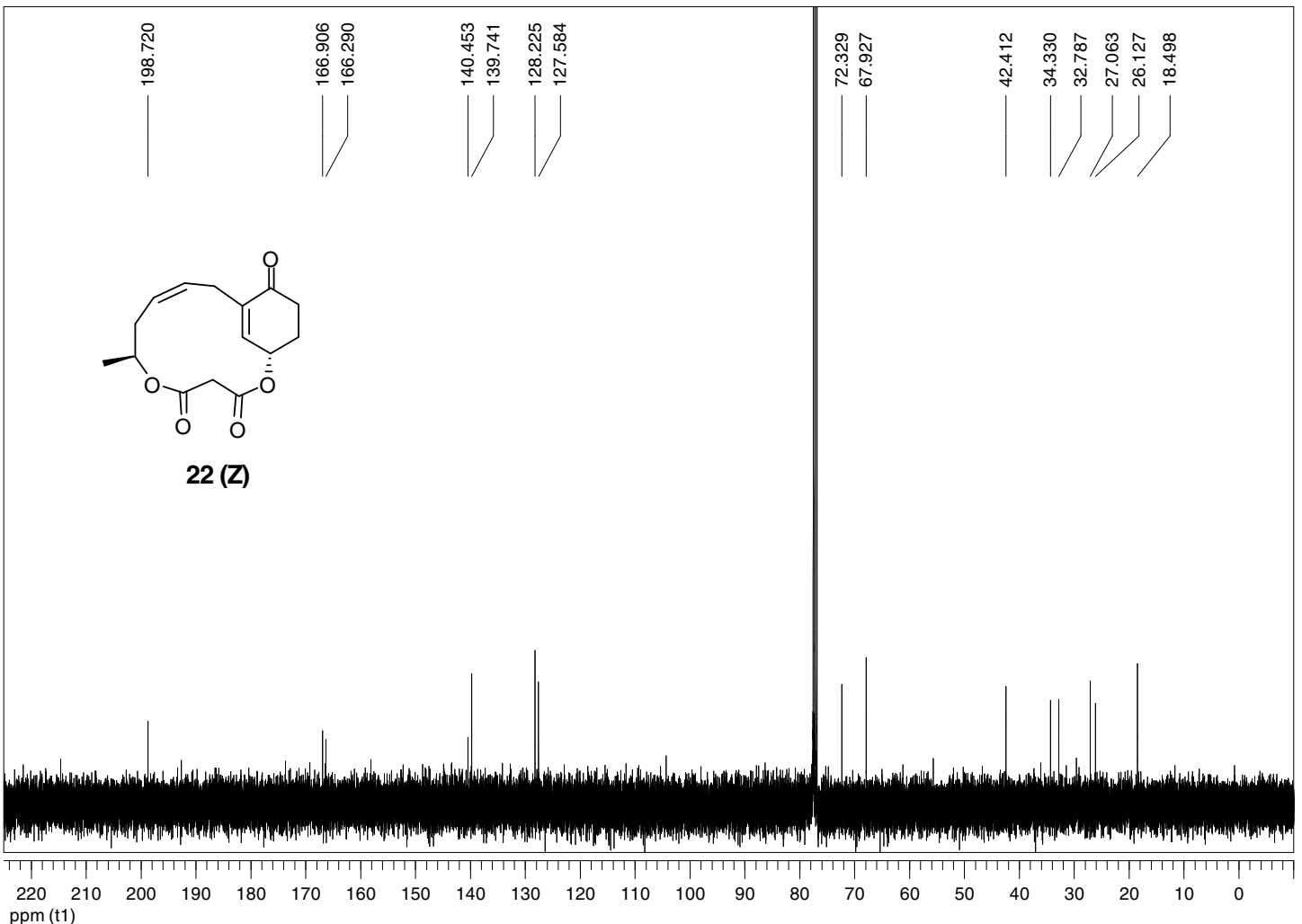




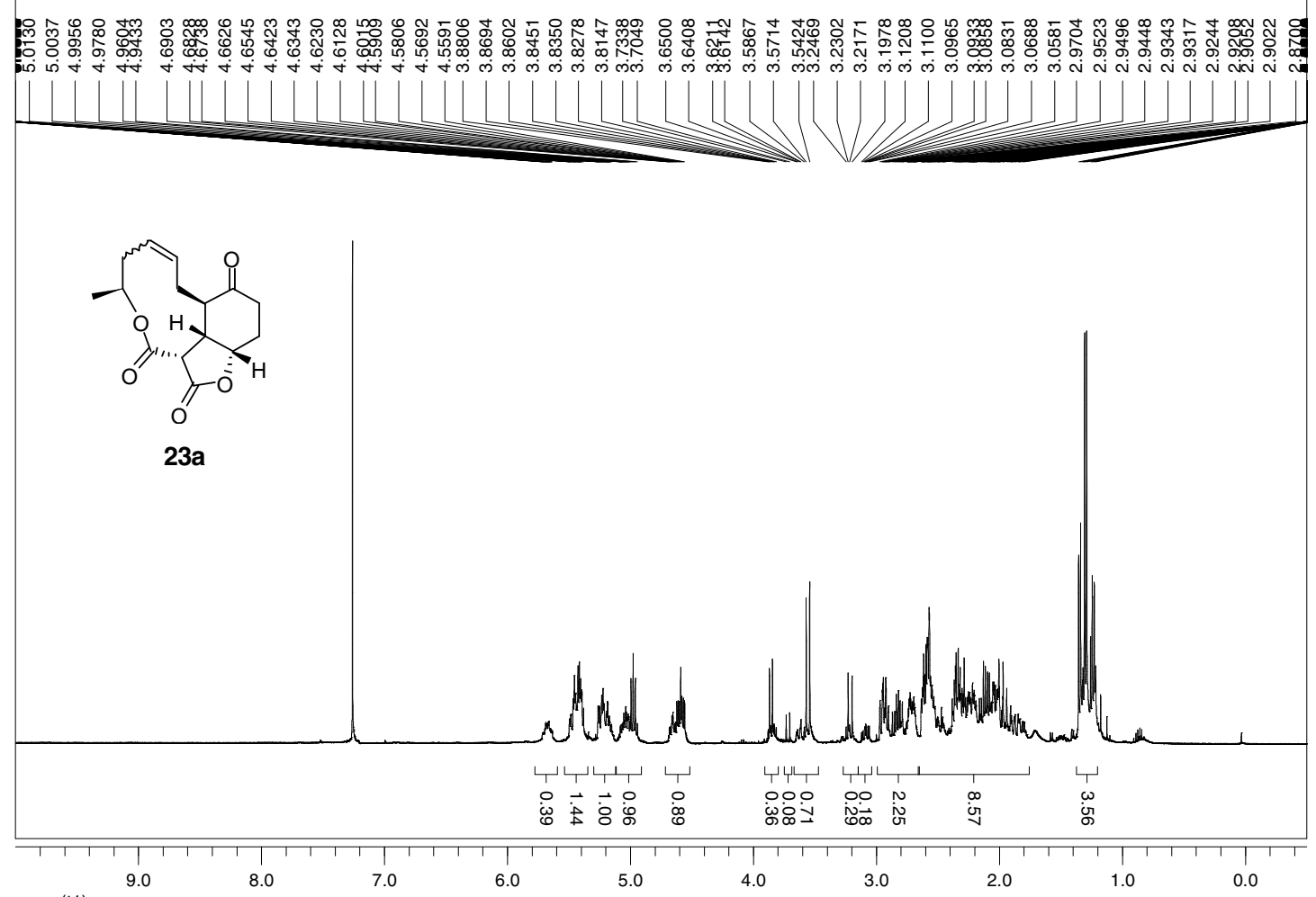

ppm (t1)

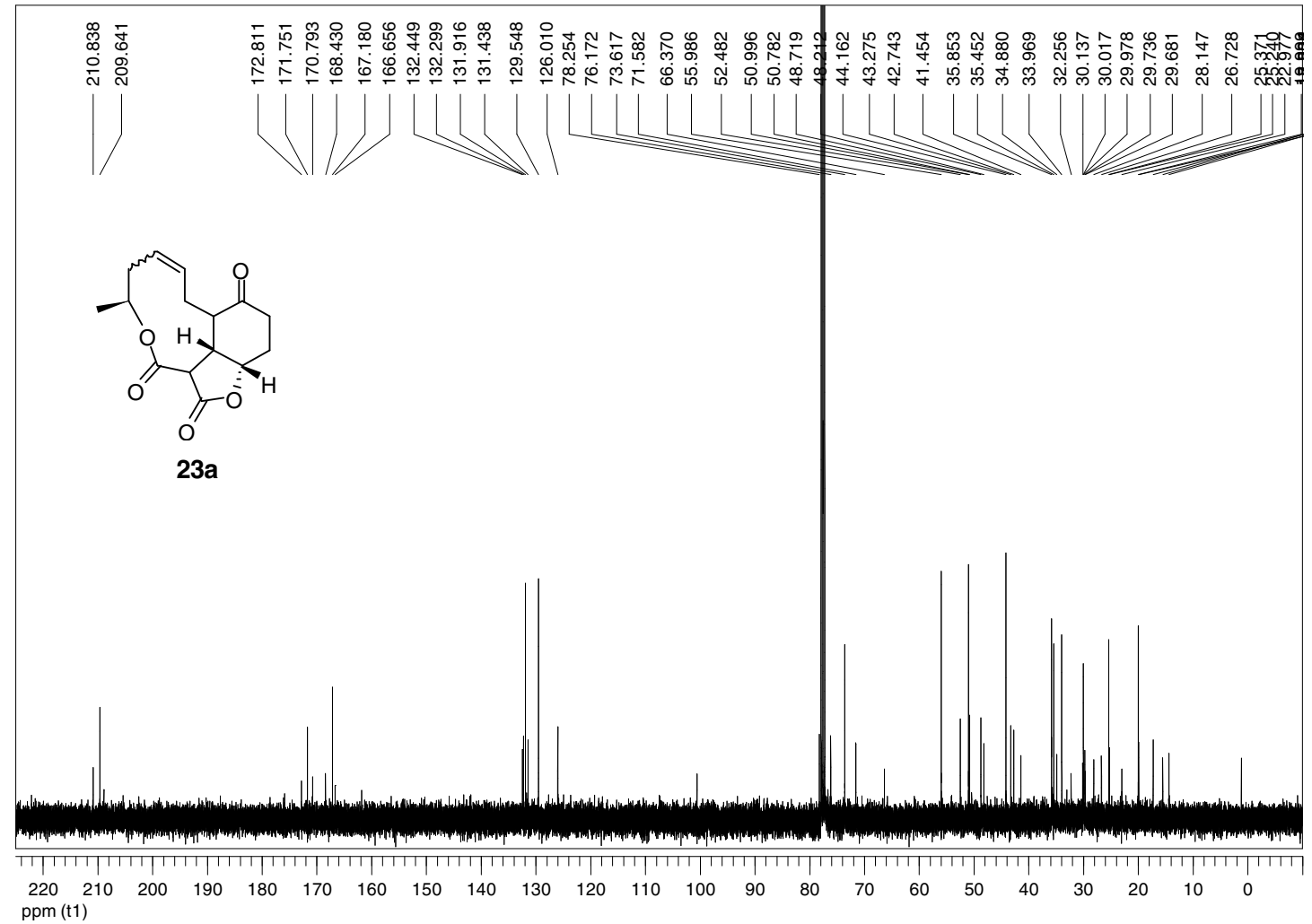




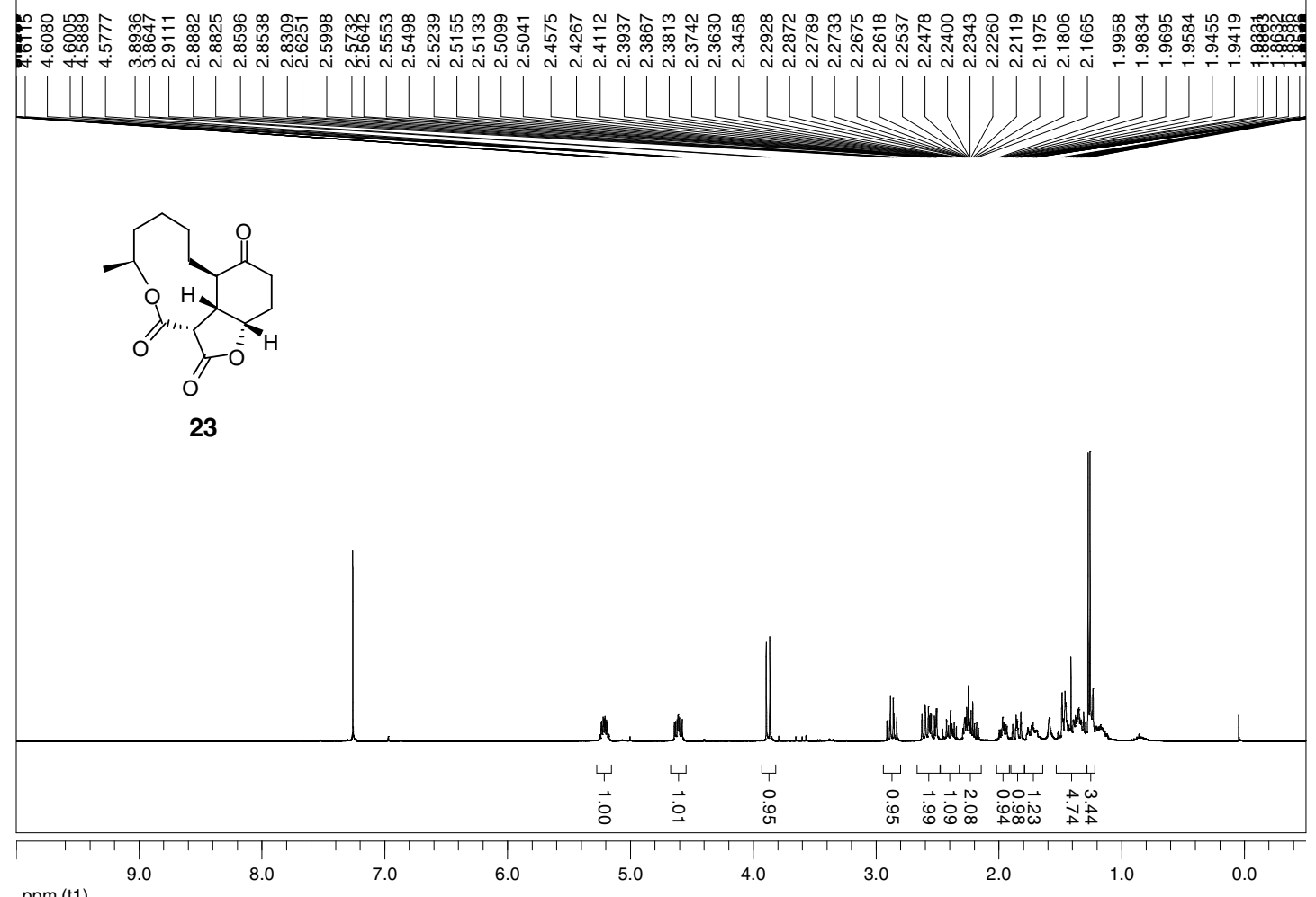

ppm (t1)
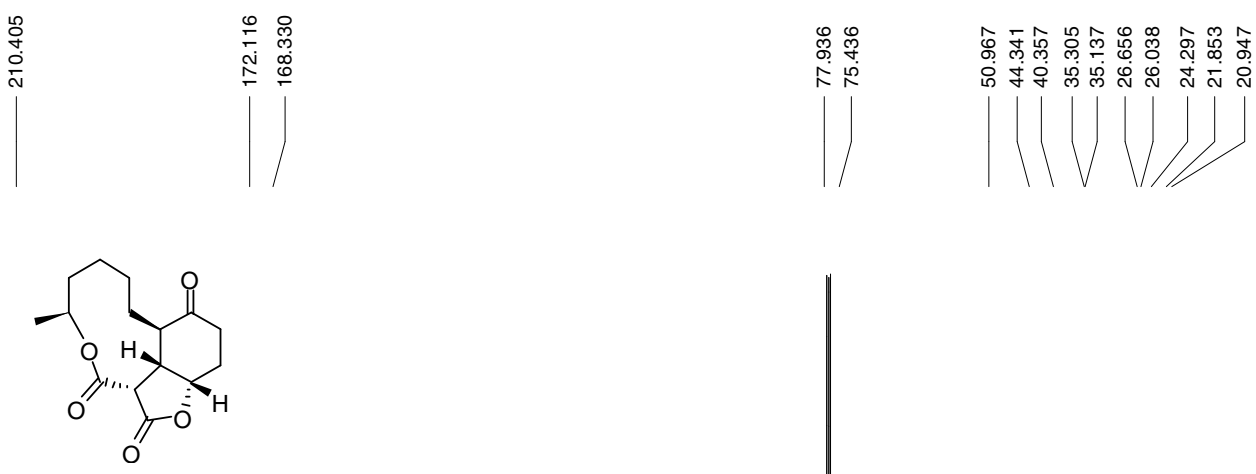

23 


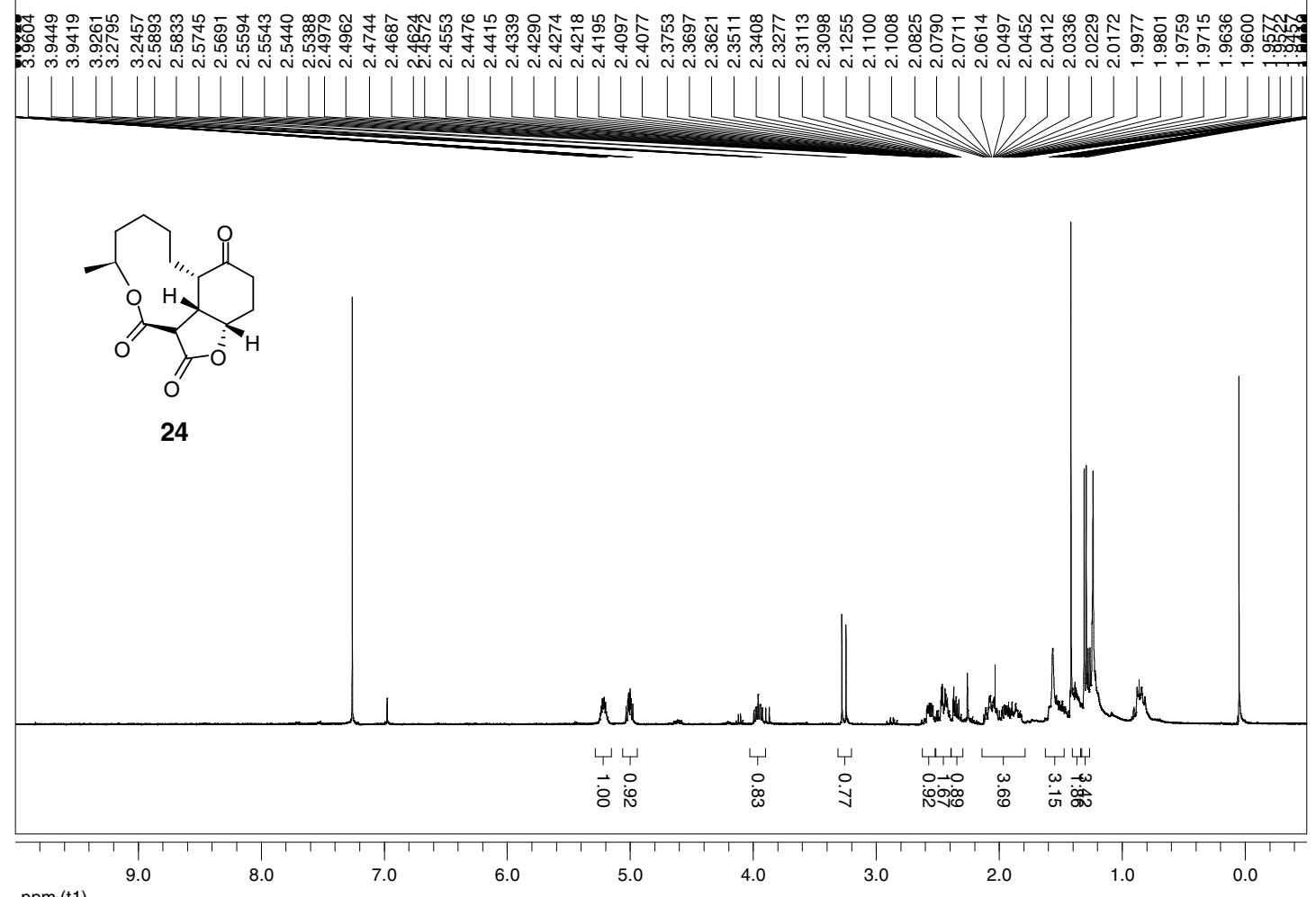

ppm (t1)

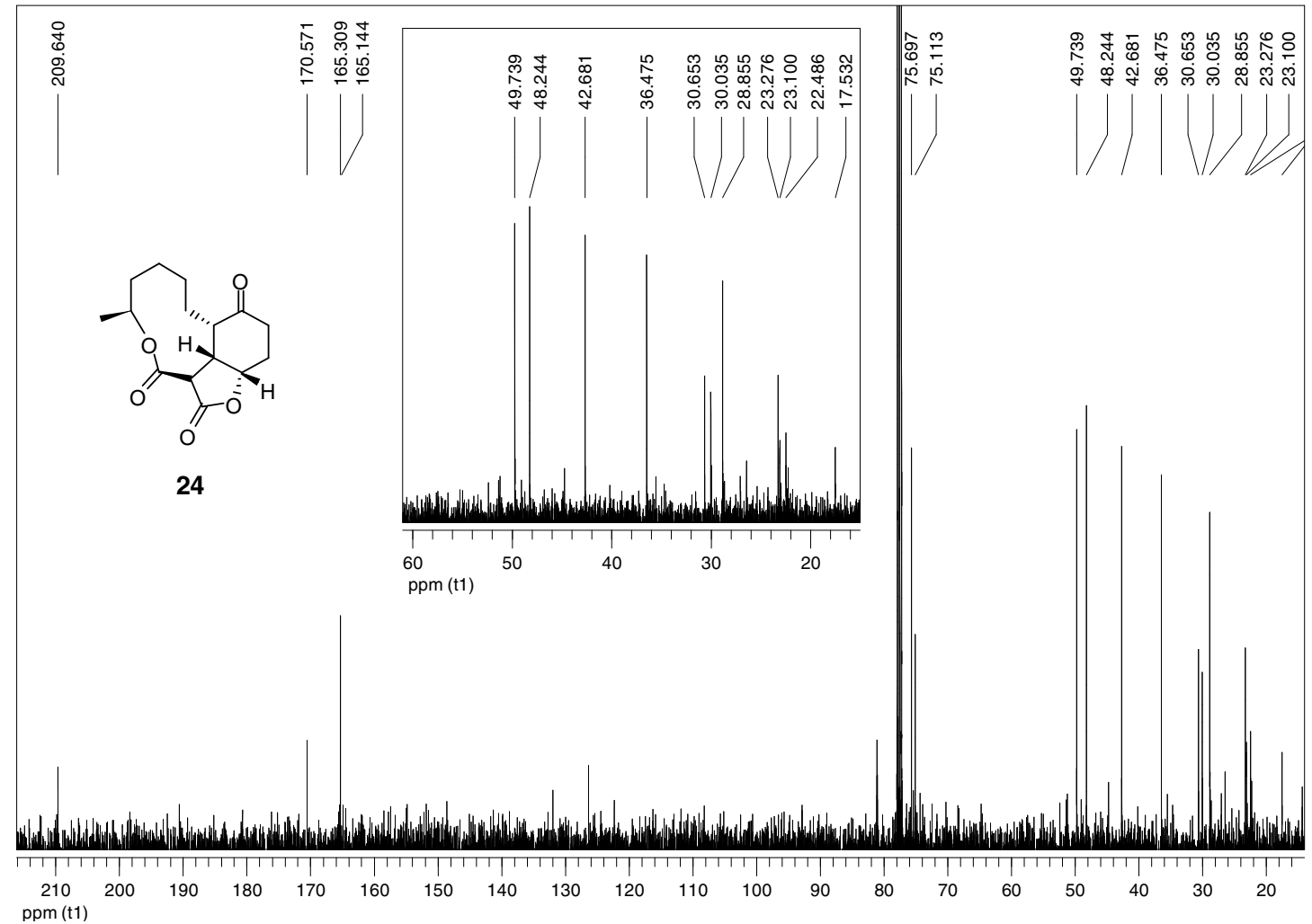




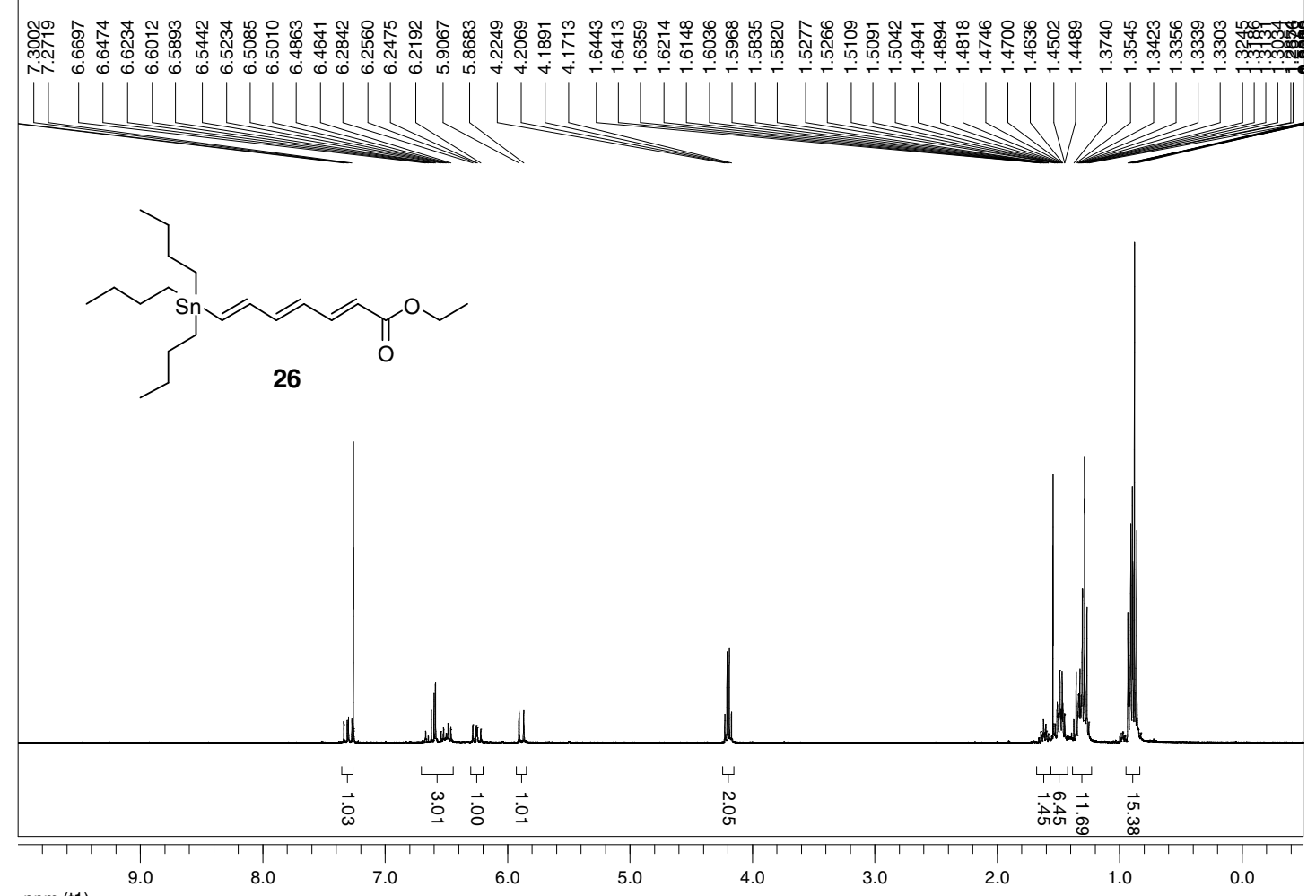

ppm (t1)

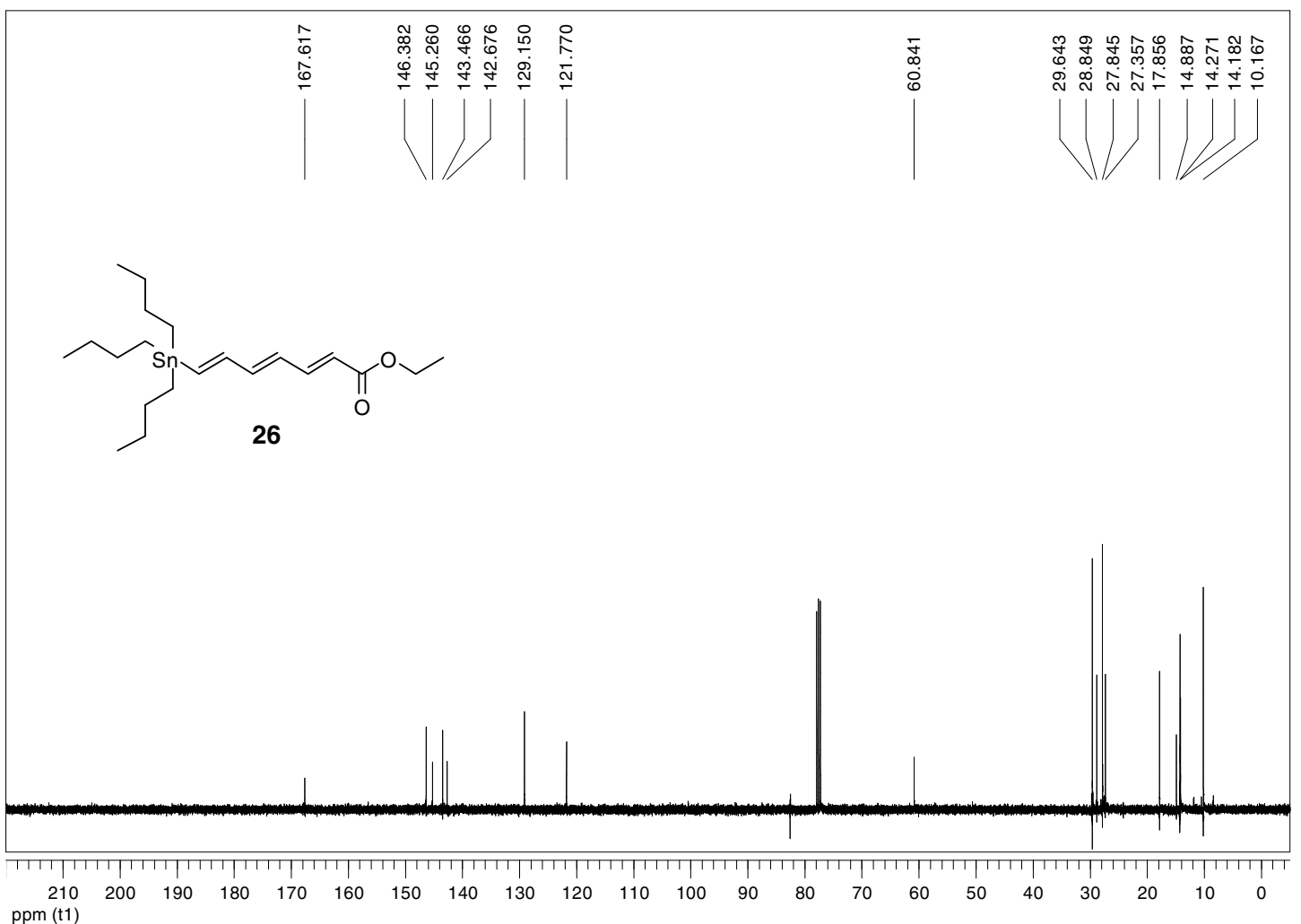




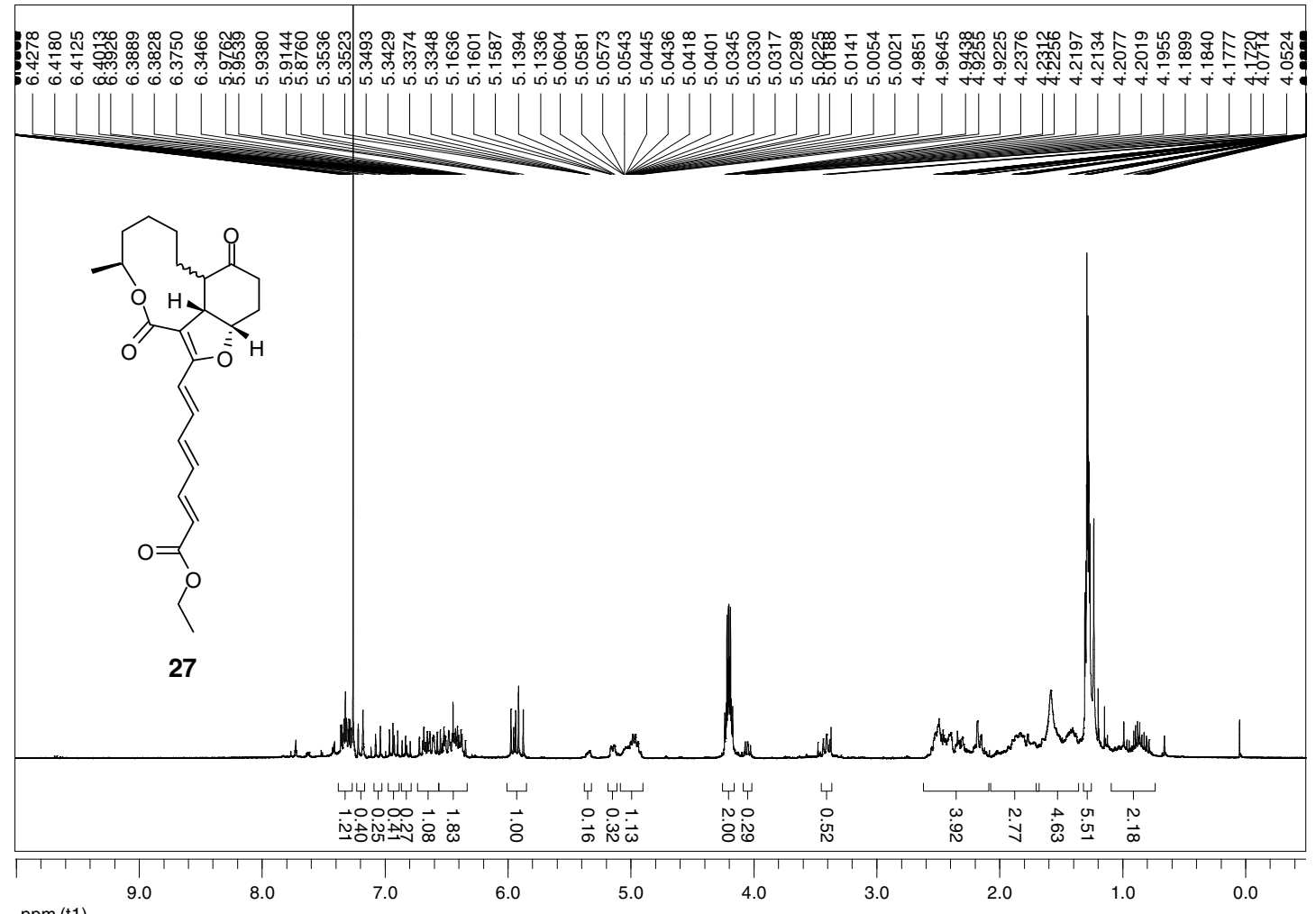
ppm (t1)

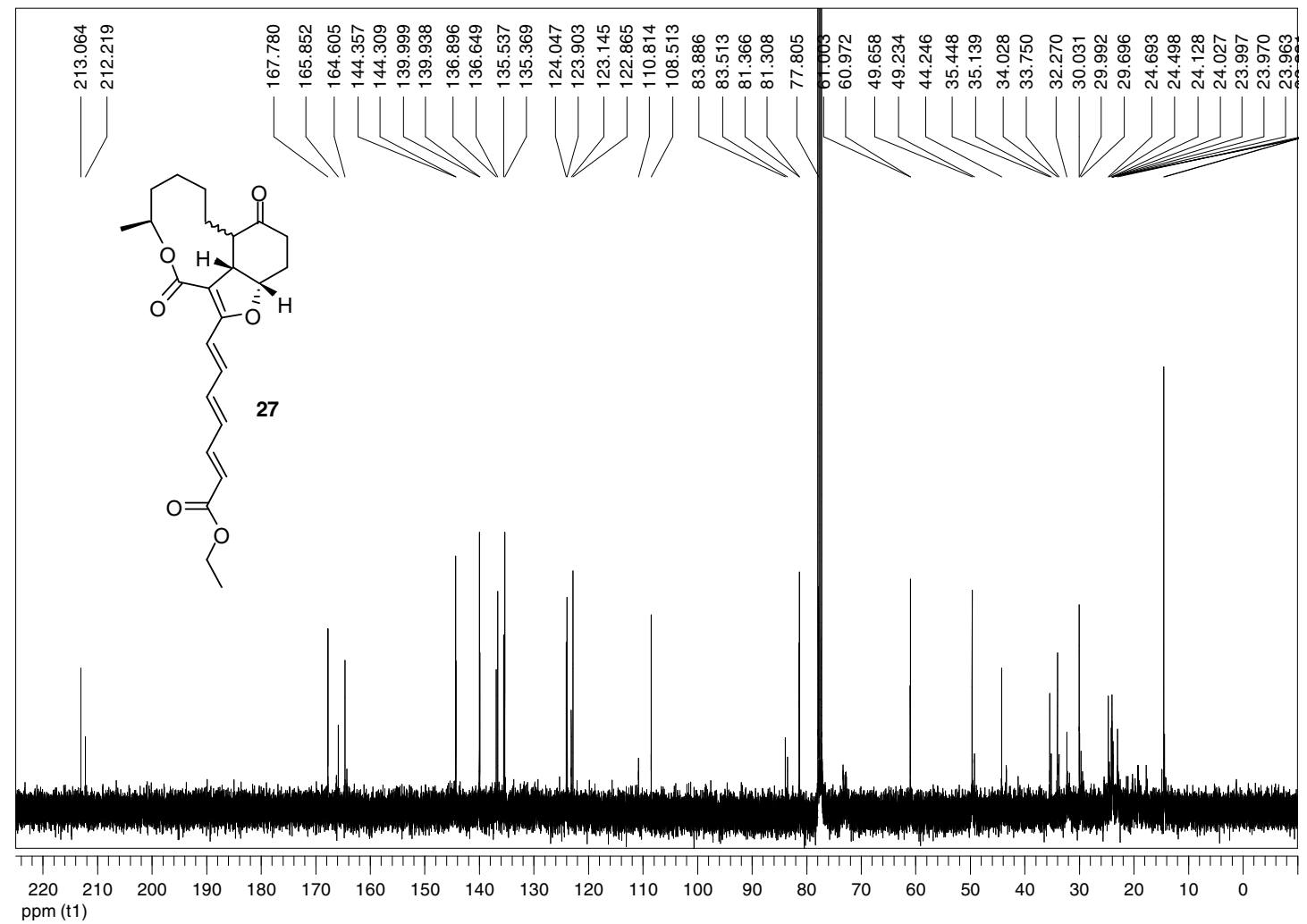




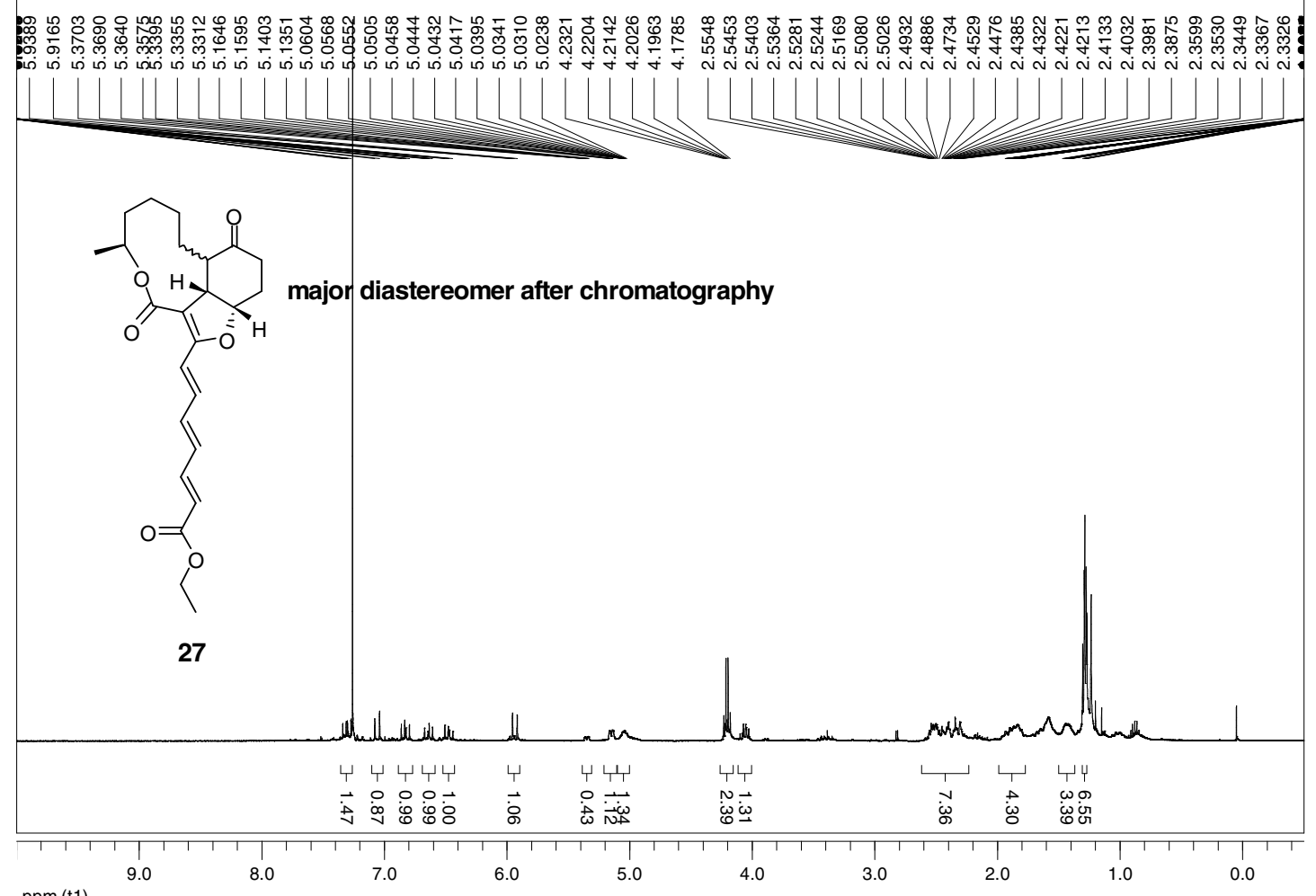

ppm (t1) 